\title{
Scattering equations: from projective spaces to tropical grassmannians
}

\author{
Freddy Cachazo, ${ }^{a}$ Nick Early, ${ }^{b}$ Alfredo Guevara ${ }^{a, c, d}$ and Sebastian Mizera ${ }^{a, c}$ \\ ${ }^{a}$ Perimeter Institute for Theoretical Physics, \\ Waterloo, ON N2L 2Y5, Canada \\ ${ }^{b}$ Massachusetts Institute of Technology, \\ Cambridge, MA, U.S.A. \\ ${ }^{c}$ Department of Physics $\&$ Astronomy, University of Waterloo, \\ Waterloo, ON N2L 3G1, Canada \\ ${ }^{d}$ CECs Valdivia \& Departamento de Física, Universidad de Concepción, \\ Casilla 160-C, Concepción, Chile \\ E-mail: fcachazo@pitp.ca, earlnick@gmail.com, aguevara@pitp.ca, \\ smizera@pitp.ca
}

ABSTRACT: We introduce a natural generalization of the scattering equations, which connect the space of Mandelstam invariants to that of points on $\mathbb{C P}^{1}$, to higher-dimensional projective spaces $\mathbb{C P}^{k-1}$. The standard, $k=2$ Mandelstam invariants, $s_{a b}$, are generalized to completely symmetric tensors $\mathbf{s}_{a_{1} a_{2} \ldots a_{k}}$ subject to a 'massless' condition $\mathbf{s}_{a_{1} a_{2} \cdots a_{k-2} b b}=0$ and to 'momentum conservation'. The scattering equations are obtained by constructing a potential function and computing its critical points. We mainly concentrate on the $k=3$ case: study solutions and define the generalization of biadjoint scalar amplitudes. We compute all 'biadjoint amplitudes' for $(k, n)=(3,6)$ and find a direct connection to the tropical Grassmannian. This leads to the notion of $k=3$ Feynman diagrams. We also find a concrete realization of the new kinematic spaces, which coincides with the spinor-helicity formalism for $k=2$, and provides analytic solutions analogous to the MHV ones.

KEYWords: Scattering Amplitudes, Differential and Algebraic Geometry

ARXIV EPRINT: 1903.08904 


\section{Contents}

1 Introduction: generalizing the potential function 1

2 Scattering equations on $\mathbb{C P}^{2}$ : Jacobians and amplitudes 4

2.1 Jacobian matrix 5

2.2 Generalized biadjoint amplitudes 5

2.2.1 Case I: $k=3$ and $n=5 \quad 6$

2.2.2 Case II: $k=3$ and $n=6 \quad 7$

2.3 Geometric interpretation 9

$\begin{array}{ll}\text { 2.3.1 Two or three punctures colliding } & 10\end{array}$

2.3.2 Three or four punctures becoming collinear 11

$\begin{array}{ll}\text { 2.3.3 Two punctures colliding on a line } & 11\end{array}$

3 Duality between $X(k, n)$ and $X(n-k, n) \quad 11$

4 Tropical Grassmannians and higher- $k$ Feynman diagrams 13

5 Matrix kinematics and MHV sectors $\quad 16$

$\begin{array}{lll}5.1 \text { Analytic solutions to the scattering equations } & 18\end{array}$

$\begin{array}{llr}6 & \text { Positive kinematics } & 20\end{array}$

$\begin{array}{lll}7 & \text { Discussion } & 23\end{array}$

$\begin{array}{ll}\text { A Euler characteristic of } X(3,6) & 25\end{array}$

B Soft limits and numbers of solutions $\quad 26$

C Numerical solution to the $X(3,6)$ scattering equations 28

\section{Introduction: generalizing the potential function}

Scattering equations connect the space of Mandelstam invariants of $n$ massless particles to that of $n$ points on $\mathbb{C P}^{1}[1]$. They can be obtained as the conditions for finding the critical points of the "potential" function

$$
\mathcal{S}:=\sum_{1 \leq a<b \leq n} s_{a b} \log (a b)
$$


Here $s_{a b}$ are known as Mandelstam invariants and $(a b)$ denotes the $\mathrm{SL}(2, \mathbb{C})$-invariant combination of the homogeneous variables of points $a$ and $b$. More explicitly,

$$
\left(\begin{array}{ll}
a & b)
\end{array}\right)=\left|\begin{array}{cc}
\sigma_{a, 1} & \sigma_{b, 1} \\
\sigma_{a, 2} & \sigma_{b, 2}
\end{array}\right| \text {. }
$$

For $\mathcal{S}$ to be a function of points on $\mathbb{C P}^{1}$, it has to be invariant under the equivalence relation of projective space $\left(\sigma_{a, 1}, \sigma_{a, 2}\right) \sim t_{a}\left(\sigma_{a, 1}, \sigma_{a, 2}\right)$ for every $a$. This is achieved provided

$$
\sum_{\substack{b=1 \\ b \neq a}}^{n} s_{a b}=0 \quad \forall a .
$$

These conditions have the physical interpretation of momentum conservation. Note that we have not used the diagonal components $s_{a a}$, which in physical applications are taken to be zero for massless particles.

Working in inhomogeneous coordinates $x_{a}:=\sigma_{a, 2} / \sigma_{a, 1}$ critical points of $\mathcal{S}$ are found by requiring

$$
\frac{\partial \mathcal{S}}{\partial x_{a}}=\sum_{\substack{b=1 \\ b \neq a}}^{n} \frac{s_{a b}}{x_{a}-x_{b}}=0 \quad \forall a,
$$

which are known as the scattering equations [1].

The scattering equations are at the heart of the Cachazo-He-Yuan (CHY) formulation $[2,3]$ of scattering amplitudes of a large variety of theories and have made manifest properties such as the Kawai-Lewellen-Tye (KLT) [4] and Bern-Carrasco-Johansson (BCJ) relations [5]. This is one of the motivations for finding natural generalizations. Moreover, generalizations to spaces other than $\mathbb{C P}^{1}$ can help in better understanding the original equations and in finding new physical applications.

For instance, a large generalization of scattering equations was introduced by one of the authors in [6] and later used to study algebraic properties of multi-loop Feynman integrals [7, 8]. Earlier exploration into constructing a higher "Möbius spin" extension of scattering equations was taken in [9].

In this work we consider another natural generalization: from points on $\mathbb{C P}^{1}$ to points on $\mathbb{C P}^{k-1}$. The standard case has been chosen to correspond to $k=2$ for historical reasons. In physical applications the scattering equations are used to integrate functions on the moduli space known as Parke-Taylor functions [10], which are the simplest examples of what are known as leading singularities [11]. In recent work [12], we considered generalization of leading singularities from $\mathbb{C P}^{1}$ to higher-dimensional projective spaces in terms of socalled $\Delta$-algebras. Such higher- $k$ leading singularities were first introduced by Franco et al. [13] and can be used to construct general non-planar on-shell diagrams in $\mathcal{N}=4$ super Yang-Mills [14].

On $\mathbb{C P}^{k-1}$ the corresponding $\mathrm{SL}(k, \mathbb{C})$ invariants are determinants of the homogeneous coordinates of $k$ points $\left(a_{1}, a_{2}, \ldots, a_{k}\right)$. It is then natural to introduce the potential function

$$
\mathcal{S}_{k}:=\sum_{1 \leq a_{1}<a_{2} \cdots<a_{k} \leq n} \mathrm{~s}_{a_{1} a_{2} \cdots a_{k}} \log \left(a_{1}, a_{2}, \ldots, a_{k}\right) .
$$


Once again, requiring $\mathcal{S}_{k}$ to be independent of the scaling of each point imposes conditions on the generalized Mandelstam invariants $\mathrm{s}_{a_{1} a_{2} \cdots a_{k}}$. It is easiest to express the condition by completing $\mathrm{s}_{a_{1} a_{2} \cdots a_{k}}$ into a symmetric rank $k$ tensor,

$$
\sum_{\substack{a_{2}, a_{3}, \ldots, a_{k}=1 \\ a_{i} \neq a_{j}}}^{n} \mathrm{~s}_{a_{1} a_{2} \cdots a_{k}}=0 \quad \forall a_{1} .
$$

It is tempting to think about the tensor $s_{a_{1} a_{2} \cdots a_{k}}$ as the multiparticle generalization of $s_{a b}$ given by the norm of the sum over the corresponding momentum vectors. As it turns out, while multiparticle invariants satisfy (1.6), they are not the most general solution and therefore we do not specialize to them as doing so would lead to singular configurations. In order to make this manifest we use a different font. Of course when $k=2$ we can write $\mathrm{s}_{a_{1} a_{2}}=s_{a_{1} a_{2}}$.

The $\mathbb{C P}^{k-1}$ scattering equations are then given by the conditions for finding critical points of $\mathcal{S}_{k}$,

$$
\frac{\partial \mathcal{S}_{k}}{\partial x_{a}^{(i)}}=0 \quad \forall(a, i)
$$

where $x_{a}^{(i)}$ represent inhomogeneous coordinates of the puncture $a$ on $\mathbb{C P}^{k-1}$. In this work we initiate the study of these equations.

We denote the moduli space on which the scattering equations (1.7) are defined by $X(k, n)$. It can be written as a quotient of the Grassmannian $G(k, n)$ by the $n$-torus action,

$$
X(k, n):=G(k, n) /\left(\mathbb{C}^{*}\right)^{n} .
$$

Since the diagonal torus action is redundant, the complex dimension of $X(k, n)$ turns out to be $(k-1)(n-k-1)$. Understanding the boundary structure of $X(k, n)$ in general proves to be a difficult mathematical problem [15-22]. Hence we focus mainly on the case $k=3$ with $n \leq 6$.

We start in section 2 by defining the analogues of the CHY formulae for $k=3$ and computing the associated "amplitudes" based on a natural generalization of Parke-Taylor factors. We find that they are rational functions with poles in the kinematic invariants $\mathrm{s}_{i j k}$, as well as more complicated linear combinations of $\mathrm{s}_{i j k}$. We then proceed by identifying singular configurations of points on $\mathbb{C P}^{2}$ associated to these kinematic poles, for example when multiple points become simultaneously collinear. In section 3 we show that there exists a duality between scattering equations on $X(k, n)$ and $X(n-k, n)$, which follows from the corresponding duality on the Grassmannian.

In the case of $k=3$ we find a surprising relation to the so-called tropical Grassmannian [23], which seems to govern the space of kinematic singularities allowed by the CHY formalism on $X(3,6)$. This allows us to associate a set of "Feynman diagrams" to each planar ordering $\alpha$ of 6 labels, and understand generalizations of biadjoint scalar amplitudes $m_{6}^{(3)}(\alpha \mid \beta)$ as a sum over the diagrams compatible with both permutations $\alpha$ and $\beta$ at the same time. This is described in section 4 . 
In section 5 we provide an interpretation of the generalized kinematic invariants $\mathrm{s}_{a_{1} a_{2} \cdots a_{k}}$ as coming from variables similar to the standard spinor-helicity formalism for $k=2$ in four dimensions. Using this kinematics we are able to prove that for any $k$ and $n$ there exist special classes of analytic solutions to the scattering equations akin to the $\mathrm{MHV}$ and $\overline{\mathrm{MHV}}$ sectors in four dimensions.

In [24] it was shown that there exist large kinematic regions in which all the solutions of the standard scattering equations are real and bounded. We generalize this construction to $k=3$ cases by interpreting (the real part of) $\mathcal{S}_{3}$ from (1.5) as a potential for interacting particles on $\mathbb{R P}^{2}$. We discuss limitations of this procedure in predicting the number of solutions of the general- $k$ scattering equations due to the fact that different soft kinematic regimes are separated by new singularities appearing at $k=3$.

We conclude with a discussion of results and future directions in section 7 . We also include two appendices. In appendix A we prove the number of solutions to the scattering equation by computing the Euler characteristic of $X(3,6)$, while in appendix B we give a lower bound for the number of solutions for general $n$ using soft limits.

\section{Scattering equations on $\mathbb{C P}^{2}$ : Jacobians and amplitudes}

In this section we specialize to $k=3$ in order to carry out explicit computations and build intuition by developing some of the same tools already known for $k=2$.

Let us simplify the notation by denoting inhomogeneous coordinates on $\mathbb{C P}^{2}$ by $(x, y)$. This means that the potential function is

$$
\mathcal{S}_{3}=\sum_{1 \leq a<b<c \leq n} \mathbf{s}_{a b c} \log \left|\begin{array}{ccc}
1 & 1 & 1 \\
x_{a} & x_{b} & x_{c} \\
y_{a} & y_{b} & y_{c}
\end{array}\right| .
$$

The scattering equations are then

$$
\sum_{\substack{1 \leq b<c \leq n \\ b, c \neq a}} \frac{\mathrm{s}_{a b c}\left(x_{b}-x_{c}\right)}{|a b c|}=0, \quad \sum_{\substack{1 \leq b<c \leq n \\ b, c \neq a}} \frac{\mathrm{s}_{a b c}\left(y_{b}-y_{c}\right)}{|a b c|}=0, \quad \forall a .
$$

Here we have introduced the shorthand notation $|a b c|$ for the determinant in $\mathcal{S}_{3}$.

At first sight, these are $2 n$ equations for $2 n$ variables. However, as it is familiar in the $k=2$ case, these equations are covariant under $\mathrm{SL}(3, \mathbb{C})$ transformations which is the automorphism group of $\mathbb{C P}^{2}$. This means that 8 equations are redundant and that we can use the group to fix the positions of 4 points (each having two coordinates) to generic, i.e., non-collinear, positions.

This makes it clear that $n \geq 4$ in order to have a stable $\mathbb{C P}^{2}$, i.e., one in which all the automorphism group is fixed. Recall that when $k=2$, this is equivalent to the statement that at least three points are needed in order to have a well-defined set of equations and develop the CHY formalism. 


\section{$2.1 \quad$ Jacobian matrix}

The next key ingredient in the CHY formalism of scattering amplitudes is the Jacobian matrix associated with the system of scattering equations. Once again, let us review the $k=2$ case before discussing the $k=3$ case.

The Jacobian matrix is a $n \times n$ symmetric matrix with components $\Phi_{a b}^{(2)}:=$ $\partial^{2} \mathcal{S}_{2} / \partial x_{a} \partial x_{b}$. It is well-known that this matrix has rank $n-3$ due to the $\operatorname{SL}(2, \mathbb{C})$ action, and therefore one has to defined a reduced determinant

$$
\operatorname{det}^{\prime} \Phi^{(2)}:=\frac{\operatorname{det} \Phi_{p q r}^{(2) i j k}}{V_{i j k} V_{p q r}},
$$

where the Vandermonde determinants are defined as $V_{i j k}:=\left(x_{i}-x_{j}\right)\left(x_{j}-x_{k}\right)\left(x_{k}-x_{i}\right)$ and $\Phi_{p q r}^{(2) i j k}$ is a $(n-3) \times(n-3)$ matrix obtained by deleting rows $i, j, k$ and columns $p, q, r$ of $\Phi$. It is easy to show that the right-hand side is independent of the choice of rows and columns to delete and this is why $\operatorname{det}^{\prime} \Phi^{(2)}$ is well-defined.

Moving on to $k=3$, one has that the Jacobian matrix is a $2 n \times 2 n$ matrix with a block structure. The $n \times n$ blocks have components $\Phi_{a b}^{(3)}:=\partial^{2} \mathcal{S}_{3} / \partial x_{a} \partial x_{b}, \Phi_{n+a, n+b}^{(3)}:=$ $\partial^{2} \mathcal{S}_{3} / \partial y_{a} \partial y_{b}, \Phi_{a, n+b}^{(3)}:=\partial^{2} \mathcal{S}_{3} / \partial x_{a} \partial y_{b}$, and $\Phi_{n+a, b}^{(3)}:=\partial^{2} \mathcal{S}_{3} / \partial y_{a} \partial x_{b}$.

Once again the matrix is singular and it has rank $2 n-8$ due to the $\mathrm{SL}(3, \mathbb{C})$ action. The reduced determinant in this case is obtained in a completely analogous manner,

$$
\operatorname{det}^{\prime} \Phi^{(3)}:=\frac{\operatorname{det} \Phi_{p q r s}^{(3) i j k l}}{V_{i j k l} V_{p q r s}}
$$

where $\Phi_{\text {pqrs }}^{(3) i j k l}$ is the matrix obtained from $\Phi^{(3)}$ by deleting rows $\{i, i+n, j, j+n, k, k+n, l, l+n\}$ and columns $\{p, p+n, q, q+n, r, r+n, s, s+n\}$. The generalized Vandermonde factors are defined as

$$
V_{i j k l}:=|i j k||j k l||k l i||l i j| .
$$

\subsection{Generalized biadjoint amplitudes}

The simplest scattering amplitudes that admit a CHY representation are those of the biadjoint scalar theory [3]. In this $k=2$ construction the amplitudes depend on the choice of two cyclic orderings $\alpha$ and $\beta$ and are computed as

$$
m_{n}^{(k=2)}(\alpha \mid \beta)=\frac{1}{\operatorname{vol}(\mathrm{SL}(2, \mathbb{C}))} \int \prod_{a=1}^{n} d x_{a} \prod_{a=1}^{n} \delta\left(\frac{\partial \mathcal{S}_{2}}{\partial x_{a}}\right) \mathrm{PT}^{(2)}(\alpha) \mathrm{PT}^{(2)}(\beta)
$$

where Parke-Taylor functions are defined as

$$
\mathrm{PT}^{(2)}(12 \cdots n):=\frac{1}{\left(x_{1}-x_{2}\right)\left(x_{2}-x_{3}\right) \cdots\left(x_{n}-x_{1}\right)}
$$

and the pre-factor containing $\operatorname{vol}(\mathrm{SL}(2, \mathbb{C}))$ is there to indicate that the integral as such is not well-defined and the $\operatorname{SL}(2, \mathbb{C})$ redundancy has to be fixed. This is done by fixing 
three coordinates, say $x_{i}, x_{j}, x_{k}$ and removing three delta functions, say $p, q, r$. There is a Fadeev-Popov factor that is generated and it is given by $V_{i j k} V_{p q r}$.

Explicitly evaluating the formula on the solutions $x_{a}^{(I)}$ to the scattering equations gives

$$
m_{n}^{(2)}(\alpha \mid \beta)=\left.\sum_{I=1}^{(n-3) !}\left(\frac{1}{\operatorname{det}^{\prime} \Phi^{(2)}} \mathrm{PT}^{(2)}(\alpha) \mathrm{PT}^{(2)}(\beta)\right)\right|_{x_{a}=x_{a}^{(I)}} .
$$

The simplest amplitude is for $n=3$. In that case $\operatorname{det}^{\prime} \Phi^{(2)}=1 / V_{123}^{2}$ and $m_{3}^{(2)}(\alpha \mid \beta)=1$.

The first non-trivial amplitude is for $n=4$. A simple computation reveals that, e.g.,

$$
m_{4}^{(2)}(1234 \mid 1234)=\frac{1}{s_{12}}+\frac{1}{s_{23}} .
$$

Let us also give a result for $n=5$ as this will be useful in the $k=3$ discussion,

$$
m_{5}^{(2)}(12345 \mid 12345)=\frac{1}{s_{12} s_{34}}+\frac{1}{s_{23} s_{45}}+\frac{1}{s_{34} s_{51}}+\frac{1}{s_{45} s_{12}}+\frac{1}{s_{51} s_{23}} .
$$

Now we are ready to consider $k=3$ amplitudes. In analogy with the biadjoint theory (2.6) we define

$$
m_{n}^{(3)}(\alpha \mid \beta):=\int d \mu_{3, n} \mathrm{PT}^{(3)}(\alpha) \mathrm{PT}^{(3)}(\beta),
$$

where

$$
d \mu_{3, n}:=\frac{1}{\operatorname{vol}(\operatorname{SL}(3, \mathbb{C}))} \prod_{a=1}^{n} d x_{a} d y_{a} \prod_{a=1}^{n} \delta\left(\frac{\partial \mathcal{S}_{3}}{\partial x_{a}}\right) \delta\left(\frac{\partial \mathcal{S}_{3}}{\partial y_{a}}\right)
$$

The $k=3$ Parke-Taylor functions are defined as

$$
\mathrm{PT}^{(3)}(1,2, \ldots, n):=\frac{1}{|123||234| \cdots|n 12|} .
$$

Once again, the factor $\operatorname{vol}(\mathrm{SL}(3, \mathbb{C}))$ is there to indicate that the corresponding redundancy must be fixed before attempting the integration. After fixing the redundancy one finds

$$
m_{n}^{(3)}(\alpha \mid \beta)=\left.\sum_{I=1}^{\mathcal{N}_{n}^{(3)}}\left(\frac{1}{\operatorname{det}^{\prime} \Phi^{(3)}} \mathrm{PT}^{(3)}(\alpha) \mathrm{PT}^{(3)}(\beta)\right)\right|_{x_{a}=x_{a}^{(I)}, y_{a}=y_{a}^{(I)}}
$$

Here $\mathcal{N}_{n}^{(3)}$ is the number of solutions to the scattering equations while the reduced determinant $\operatorname{det}^{\prime} \Phi^{(3)}$ is defined in (2.4). In the cases of interest in this section the number of solutions are $\mathcal{N}_{5}^{(3)}=2$ and $\mathcal{N}_{6}^{(3)}=26$. We prove this in appendix A.

Let us illustrate the definition with examples. Clearly, $m_{4}^{(3)}(\alpha \mid \beta)=1$. The first nontrivial amplitudes are for $n=5$. We start with this case and then move on to $n=6$.

\subsubsection{Case I: $k=3$ and $n=5$}

Explicit computations show that

$$
m_{5}^{(3)}(\alpha \mid \beta)=\left.m_{5}^{(2)}(\alpha \mid \beta)\right|_{s_{a b} \rightarrow \mathbf{s}_{c d e}}
$$

where $\{a, b, c, d, e\}=\{1,2,3,4,5\}$. 
For example,

$$
m_{5}^{(3)}(12345 \mid 12345)=\frac{1}{\mathrm{~s}_{345} \mathrm{~s}_{512}}+\frac{1}{\mathrm{~s}_{451} \mathrm{~s}_{123}}+\frac{1}{\mathrm{~s}_{512} \mathrm{~s}_{234}}+\frac{1}{\mathrm{~s}_{123} \mathrm{~s}_{345}}+\frac{1}{\mathrm{~s}_{234} \mathrm{~s}_{451}} .
$$

and

$$
m_{5}^{(3)}(12345 \mid 13524)=0 .
$$

This correspondence between the $k=3, n=5$ case and the $k=2, n=5$ is not an accident. In section 3, we prove that solutions and amplitudes for $(k, n)$ map to those for $(n-k, n)$ as a consequence of the isomorphism between the Grassmannians $G(k, n)$ and $G(n-k, n)$.

\subsubsection{Case II: $k=3$ and $n=6$}

Considering $n=6$ and $k=3$ produces new objects but still with similar features to those of $n=6$ and $k=2$. We have computed several explicit examples.

The first class is the set of orderings orthogonal to the identity or canonical order, i.e., those for which the $k=2$ amplitudes vanish. As it turns out the same amplitudes vanish for $k=3$. Explicitly, introducing the notation $\mathbb{I}=123456$ for the canonical ordering, we have:

$$
m_{6}^{(3)}(\mathbb{I} \mid 125364)=m_{6}^{(3)}(\mathbb{I} \mid 124635)=m_{6}^{(3)}(\mathbb{I} \mid 136425)=0 .
$$

The second class is that of orderings for which only one Feynman diagram contributes to the $k=2$ case:

$$
m_{6}^{(3)}(\mathbb{I} \mid 126435)=\frac{1}{\mathrm{~s}_{612} \mathrm{~s}_{345}\left(\mathrm{~s}_{345}+\mathrm{s}_{346}+\mathrm{s}_{356}+\mathrm{s}_{456}\right)\left(\mathrm{s}_{561}+\mathrm{s}_{562}+\mathrm{s}_{512}+\mathrm{s}_{612}\right)} .
$$

There are two interesting features that deserve attention. The first is the appearance of both $\mathrm{s}_{612}$ and $\mathrm{s}_{345}$. If they were standard $k=2$ three-particle kinematic invariants momentum conservation would have equated both of them. Here however they are independent and as discussed in the next section, the presence of both poles corresponds to two different geometric configurations; one in which $6,1,2$ are collinear and the other where $3,4,5$ are collinear. The second feature is the structure of the other two poles. Note that they are the straightforward generalization of $(k+1)$-particle invariants when $k$ particle invariants are taken as fundamental. Motivated by this we define

$$
\mathrm{t}_{a_{1} a_{2} \ldots a_{k} a_{k+1}}:=\sum_{i=1}^{k+1} \mathrm{~s}_{a_{1} a_{2} \ldots \hat{a}_{i} \ldots a_{k+1}} .
$$

For example, when $k=2$ one has $t_{a b c}=s_{b c}+s_{a c}+s_{a b}$, recall that we use the standard font for both $t$ and $s$ when $k=2$. Using this notation one has

$$
m_{6}^{(3)}(\mathbb{I} \mid 126435)=\frac{1}{\mathrm{~s}_{612} \mathrm{~s}_{345} \mathrm{t}_{3456} \mathrm{t}_{5612}}
$$

and

$$
m_{6}^{(3)}(\mathbb{I} \mid 125463)=\frac{1}{\mathrm{~s}_{123} \mathrm{~s}_{456} \mathrm{t}_{6123} \mathrm{t}_{3456}}
$$


There is one more topology of $k=2$ amplitudes with a single Feynman diagram. It corresponds to the orderings $(\mathbb{I} \mid 125634)$. This turns out to introduce a new class of poles with no $k=2$ analog. This is why we first discuss other amplitudes which contain two Feynman diagrams in $k=2$ before returning to $(\mathbb{I} \mid 125634)$,

$$
\begin{aligned}
& m_{6}^{(3)}(\mathbb{I} \mid 125436)=\frac{1}{\mathrm{~s}_{345} \mathrm{~s}_{126}}\left(\frac{1}{\mathrm{t}_{2345}}+\frac{1}{\mathrm{t}_{3456}}\right)\left(\frac{1}{\mathrm{t}_{5612}}+\frac{1}{\mathrm{t}_{6123}}\right), \\
& m_{6}^{(3)}(\mathbb{I} \mid 126453)=-\frac{1}{\mathrm{t}_{3456} \mathrm{t}_{1236}}\left(\frac{1}{\mathrm{~s}_{345}}+\frac{1}{\mathrm{~s}_{456}}\right)\left(\frac{1}{\mathrm{~s}_{612}}+\frac{1}{\mathrm{~s}_{123}}\right), \\
& m_{6}^{(3)}(\mathbb{I} \mid 145623)=\frac{1}{\mathrm{t}_{1456} \mathrm{~s}_{123} \mathrm{~s}_{456}}\left(\frac{1}{\mathrm{t}_{6123}}+\frac{1}{\mathrm{t}_{1234}}\right) .
\end{aligned}
$$

Let us now turn to $m_{6}^{(3)}(\mathbb{I} \mid 125634)$ to study the new feature of $k=3$ amplitudes. Explicit computation reveals that

$$
m_{6}^{(3)}(\mathbb{I} \mid 125634)=\frac{\mathrm{t}_{1234}+\mathrm{t}_{3456}+\mathrm{t}_{5612}}{\mathrm{t}_{1234} \mathrm{t}_{3456} \mathrm{t}_{5612} R \tilde{R}},
$$

where both $R$ and $\tilde{R}$ are polynomials of degree one in the kinematic invariants and are given by

$$
R=\mathrm{s}_{123}+\mathrm{s}_{124}+\mathrm{s}_{125}+\mathrm{s}_{126}+\mathrm{s}_{134}+\mathrm{s}_{234}, \quad \tilde{R}=R_{(12) \leftrightarrow(34)} .
$$

Alternatively, we can denote both combinations by $R_{12,34,56}$ and $R_{34,12,56}$. This notation exhibits the symmetry of the object under cyclic shift of these pairs, i.e.,

$$
R=R_{12,34,56}=R_{34,56,12}=R_{56,12,34}
$$

These identities require the use of momentum conservation.

The form of (2.26) suggest that the expression should be expanded in terms of three objects; each containing poles of the form $\mathrm{tt} R \tilde{R}$. However, it turns out that (2.26) proves to be a single object. In order to motivate this interpretation it is important to note the identity

$$
R+\tilde{R}=\mathrm{t}_{1234}+\mathrm{t}_{3456}+\mathrm{t}_{5612}
$$

This means that (2.26) can also be written as the sum over only two terms, each with a pole structure $\operatorname{ttt} R$. This 3 equals 2 identity is reminiscent of identities found by computing the "volume" of a bipyramid. The volume can be computed either by summing over the top and bottom tetrahedra or by slicing through the middle line and produce three tetrahedra, each containing the top and bottom vertices. This means that none of the two ways of computing the volume are fundamental and that the object of interest is the whole bipyramid. In section 4 we will see that this intuition is correct, which is why we introduce the notation

$$
\Theta:=\frac{\mathrm{t}_{1234}+\mathrm{t}_{3456}+\mathrm{t}_{5612}}{\mathrm{t}_{1234} \mathrm{t}_{3456} \mathrm{t}_{5612} R \tilde{R}} .
$$


This object completes the lists of possible singularities appearing in $m_{6}^{(3)}(\alpha \mid \beta)$. We can now construct other amplitudes containing two or more $k=2$ Feynman diagrams:

$$
\begin{aligned}
& m_{6}^{(3)}(\mathbb{I} \mid 125643)=-\frac{1}{\mathrm{t}_{1234} \mathrm{t}_{3456}}\left(\frac{1}{\mathrm{~s}_{456} R}+\frac{1}{\mathrm{~s}_{123} \tilde{R}}+\frac{1}{\mathrm{~s}_{123} \mathrm{~s}_{456}}\right)-\Theta, \\
& m_{6}^{(3)}(\mathbb{I} \mid 123465)=-\frac{1}{\mathrm{t}_{1234}}\left[\frac{1}{R}\left(\frac{1}{\mathrm{~s}_{234} \mathrm{~s}_{456}}+\frac{1}{\mathrm{~s}_{456} \mathrm{t}_{3456}}+\frac{1}{\mathrm{~s}_{234} \mathrm{t}_{5612}}\right)\right. \\
& +\frac{1}{\tilde{R}}\left(\frac{1}{\mathrm{~s}_{123} \mathrm{~s}_{156}}+\frac{1}{\mathrm{~s}_{123} \mathrm{t}_{3456}}+\frac{1}{\mathrm{~s}_{156} \mathrm{t}_{5612}}\right) \\
& +\frac{1}{s_{456}}\left(\frac{1}{s_{123} t_{3456}}+\frac{1}{t_{1456} s_{123}}+\frac{1}{t_{1456} s_{234}}\right) \\
& \left.+\frac{1}{s_{156}}\left(\frac{1}{s_{234} t_{1256}}+\frac{1}{t_{1456} s_{234}}+\frac{1}{t_{1456} s_{123}}\right)\right]-\Theta .
\end{aligned}
$$

There is only one more amplitude missing from the set of all possible 'biadjoint' amplitudes. The one missing is $m_{6}^{(3)}(\mathbb{I} \mid \mathbb{I})$. As it is familiar from $k=2$ amplitudes, this case is the one with the largest number of terms. We postpone its computation to section 4 where we present the analog of a Feynman diagram computation, i.e., a purely combinatorial argument which turns out to be based on an interesting connection to tropical Grassmannians.

It can be checked that all amplitudes we have presented are invariant under the exchange $\mathrm{s}_{a b c} \rightarrow \mathrm{s}_{\text {def }}$, where $\{a, b, c, d, e, f\}=\{1,2,3,4,5,6\}$. This exchanges $R$ with $\tilde{R}$ and leaves t's invariant. This property is the $n=6$ analog of the property (2.15) and, as we will see in section 3 , follows from self-duality of $G(3,6)$.

Finally, the presence of the different types of singularities in the above amplitudes hints at a richer boundary structure of $X(3, n)$ as compared to $X(2, n)$. Therefore we end this section by studying configurations of points on $\mathbb{C P}^{2}$ that give rise to these singularities.

\subsection{Geometric interpretation}

Singularity structure of the amplitudes computed with the new scattering equations is governed by the potential function $\mathcal{S}_{k}$. To be more precise, the pole locus of the logarithmic 1-form $d \mathcal{S}_{k}$ specifies the boundaries of $X(k, n)$. The only difficulty in studying these boundaries is that not all of them are accessible in the same chart of $X(k, n)$ as blow-ups might be necessary. Therefore the strategy for checking whether a given configuration of points on $\mathbb{C P}^{k-1}$ is a codimension- 1 boundary is to first change coordinates such that it is approached as $\varepsilon \rightarrow 0$ from a generic configuration, and then compute

$$
\operatorname{Res}_{\varepsilon=0}\left(d \mathcal{S}_{3}\right),
$$

which gives the corresponding factorization channel. If the above residue is zero or the change of variables is not valid then the configuration is not a codimension- 1 boundary of $X(k, n)$. Higher-codimension boundaries are easily obtained by intersecting multiple codimension-1 boundaries.

Before discussing the singularity structure of $X(3,6)$ let us consider that of $X(3,5)$, which is more familiar because of the duality to $\mathcal{M}_{0,5} \cong X(2,5)$ (as discussed in section 3 ). 
Naively, there are two types of singularities that are allowed. The first one is when two points, say $a$ and $b$, collide with each other. It can be parametrized by

$$
x_{b}=x_{a}+\varepsilon \hat{x}_{b}, \quad y_{b}=y_{a}+\varepsilon \hat{y}_{b},
$$

which gives

$$
|a b c|=\varepsilon\left(\left(x_{a} \hat{y}_{b}-y_{a} \hat{x}_{b}\right)+\left(\hat{x}_{b} y_{c}-\hat{y}_{b} x_{c}\right)\right), \quad|a c d|=|b c d|+\mathcal{O}(\varepsilon),
$$

for $c, d \neq a, b$. Fixing $\hat{x}_{b}$ and changing the variables from $\left(x_{b}, y_{b}\right)$ to $\left(\varepsilon, \hat{y}_{b}\right)$ gives

$$
d \mathcal{S}_{3}=\frac{d \varepsilon}{\varepsilon} \sum_{c \neq a, b} \mathrm{~s}_{a b c}+\mathcal{O}\left(\varepsilon^{0}\right) .
$$

Using momentum conservation we find $\sum_{c \neq a, b} \mathrm{~s}_{a b c}=\mathrm{s}_{\text {def }}$, where $\{d, e, f\}$ are the three points in the complement of $\{a, b\}$. Therefore $\mathbf{s}_{\text {def }}$ is the factorization channel associated to $x_{a}$ and $x_{b}$ colliding. More points cannot collide since there is no change of variables allowing for such a scenario.

Another option is that three points, say $d, e$ and $f$, become collinear. Clearly the only vanishing angle bracket is $\mid$ def $\mid \sim \varepsilon$, which means that

$$
d \mathcal{S}_{3}=\frac{d \varepsilon}{\varepsilon} \mathbf{s}_{d e f}+\mathcal{O}\left(\varepsilon^{0}\right),
$$

and hence this yields the same type of singularity as two complementary points colliding with each other. It is straightforward to check that two codimension-1 boundaries intersect only if their channels $\mathbf{s}_{\text {def }}$ share exactly one label, e.g., $\mathbf{s}_{123}$ and $\mathbf{s}_{145}$ are compatible, but $\mathrm{s}_{123}$ and $\mathrm{s}_{124}$ are not. This concludes the description of the boundary structure of $X(3,5)$.

Given the above discussion we move on to studying boundaries of $X(3,6)$, where we have the following classes of singularities.

\subsubsection{Two or three punctures colliding}

For $n=6$ we can have two or three punctures colliding with each other (four or more is not allowed as it would be inconsistent with $\mathrm{SL}(3, \mathbb{C})$ invariance). In the first case, when $a$ and $b$ collide, we have $|a b c| \sim \varepsilon$ for all other $c$ and hence find the channel

$$
\sum_{c \neq a, b} \mathrm{~s}_{a b c}=\mathrm{t}_{d e f g}
$$

where $\{d, e, f, g\}$ is the complement of $\{a, b\}$. In the second case, say $a, b$ and $c$ colliding at the same speed we find

$$
|a b c| \sim \varepsilon^{2}, \quad|a b d| \sim|a c d| \sim|b c d| \sim \varepsilon, \quad|a d e| \sim|b d e| \sim|c d e| \sim \varepsilon^{0}
$$

for all $d, e \neq a, b, c$. Hence the corresponding factorization channel is

$$
2 \mathrm{~s}_{a b c}+\sum_{d \neq a, b, c}\left(\mathrm{~s}_{a b d}+\mathrm{s}_{a c d}+\mathrm{s}_{b c d}\right)=\mathrm{s}_{f g h} .
$$

Note the factor of 2 in front of $\mathbf{s}_{a b c}$ in the first term due to faster vanishing of $|a b c|$. The resulting channel is simply $\mathbf{s}_{f g h}$ for the complementary set $\{f, g, h\}$ to $\{a, b, c\}$. 


\subsubsection{Three or four punctures becoming collinear}

Next we consider the singularity in which three or four points become simultaneously collinear. In the first case we only have $|a b c| \sim \varepsilon$ when $a, b, c$ become collinear and hence the singularity is simply $s_{a b c}$. Hence it is the same as the complementary three points colliding. In the second case, when $a, b, c, d$ become simultaneously collinear, we have

$$
|a b c| \sim|a b d| \sim|a c d| \sim|b c d| \sim \varepsilon,
$$

while other brackets stay finite. Hence the corresponding singularity is $\mathrm{s}_{a b c}+\mathrm{s}_{a b d}+\mathrm{s}_{a c d}+$ $\mathrm{s}_{b c d}=\mathrm{t}_{a b c d}$, which is the same as in the case of the complementary two punctures colliding.

Indeed, by applying $\mathrm{SL}(3, \mathbb{C})$ transformations one can show equivalence of the two sets of singularities.

\subsubsection{Two punctures colliding on a line}

Finally we have a codimension- 1 singularity in which two points, say $a, b$ collide with each other and at the same rate become collinear with two other punctures $c, d$. In this case we have the vanishing brackets:

$$
|a b e| \sim|a c d| \sim|b c d| \sim \varepsilon
$$

for any $e \neq a, b, c$. Hence we obtain the associated channel

$$
\sum_{e \neq a, b} \mathrm{~s}_{a b e}+\mathrm{s}_{a c d}+\mathrm{s}_{b c d}
$$

which is the $R$-type singularity: for example when $(a, b, c, d)=(1,2,3,4)$ the sum in $(2.43)$ equals to $R$ from $(2.27)$. By a change of the $\mathrm{SL}(3, \mathbb{C})$ frame this singularity is the same as $a$ colliding with $b$, while $c$ simultaneously becomes collinear with $a$ and $d$.

Using $\mathrm{SL}(3, \mathbb{C})$ transformations, we can check the invariance of the $R$-type singularity under cyclic shifts of the pairs $(a, b),(c, d),(e, f)$.

\section{Duality between $X(k, n)$ and $X(n-k, n)$}

The scattering equations inherit a duality from that of Grassmannians $G(k, n)$ and $G(n-$ $k, n)$. In order to show this it is enough to study the potential function.

Let us start with the potential function (1.5) for $n$ points on $\mathbb{C P}^{k-1}$,

$$
\mathcal{S}_{k}=\sum_{1 \leq a_{1}<a_{2}<\cdots<a_{k} \leq n} \mathrm{~s}_{a_{1} a_{2} \cdots a_{k}} \log \left(a_{1}, a_{2}, \ldots, a_{k}\right) .
$$

Combining the invariance of this function under $\operatorname{SL}(k, \mathbb{C})$ transformations and rescaling of individual points one has $\mathrm{GL}(k, \mathbb{C})$ as a subgroup. This means that we can go to a gauge fixing or frame in which the first $k \times k$ submatrix for the $k \times n$ matrix defined by the columns of the points is set to the identity. Once this is done, all the maximal minors of the matrix can be interpreted as the minors of a $(n-k) \times n$ matrix in which $\operatorname{GL}(n-k, \mathbb{C})$ has been used to set the maximal minor of the last $n-k$ columns to the identity. 
This map identifies the $k \times k$ minor $\left(a_{1}, a_{2}, \ldots, a_{k}\right)$ with the $(n-k) \times(n-k)$ minor $\left(b_{1}, b_{2}, \ldots, b_{n-k}\right)$ where the set $\left\{b_{1}, b_{2}, \ldots, b_{n-k}\right\}$ is the complement of $\left\{a_{1}, a_{2}, \ldots, a_{k}\right\}$ in $\{1,2, \ldots, n\}$ which can be denoted as $\overline{a_{1}, a_{2}, \ldots, a_{k}}$.

Applying this to $\mathcal{S}_{k}$ one finds

$$
\mathcal{S}_{k}=\sum_{1 \leq a_{1}<a_{2}<\cdots<a_{k} \leq n} \mathrm{~s}_{a_{1} a_{2} \cdots a_{k}} \log \left(\overline{a_{1}, a_{2}, \ldots, a_{k}}\right) .
$$

The goal is to show that this function can also be thought of as a general potential function for $n$ points on $\mathbb{C P}^{n-k-1}$. Given that the number of terms, $\left(\begin{array}{l}n \\ k\end{array}\right)$ also happens to be equal to $\left(\begin{array}{c}n \\ n-k\end{array}\right)$, the sum can be transformed into a sum over the complement sets by writing

$$
\mathcal{S}_{k}=\sum_{1 \leq b_{1}<b_{2}<\cdots<b_{n-k} \leq n} \mathrm{~s}_{\overline{b_{1} b_{2} \ldots b_{n-k}}} \log \left(b_{1}, b_{2}, \ldots, b_{n-k}\right) .
$$

All that remains to be shown is that the set of kinematics invariants for $\mathbb{C P}^{n-k-1}$ defined by the identification

$$
\mathrm{s}_{b_{1} b_{2} \ldots b_{n-k}}:=\mathrm{s} \overline{b_{1} b_{2} \ldots b_{n-k}}
$$

is generic and satisfies the conditions

$$
\sum_{b_{2}, \ldots, b_{n-k}} \mathrm{~s}_{b_{1} b_{2} \ldots b_{n-k}}=0, \quad \forall b_{1} \in\{1,2, \ldots, n\} .
$$

Once this is done the full $\mathrm{SL}(n-k, \mathbb{C})$ invariance can be restored.

Without loss of generality, let us choose $b_{1}=1$. Using the identification (3.4) one has to prove that

$$
\sum_{1<a_{1}<a_{2}<\cdots<a_{k} \leq n} \mathrm{~s}_{a_{1} a_{2} \ldots a_{k}}=0 .
$$

Note that the sum does not include the label 1. In order to prove this property, let us start with the conditions $\mathrm{s}_{a_{1} a_{2} \ldots a_{k}}$ are known to satisfy, (1.6), as valid kinematic variables for $n$ points on $\mathbb{C P}^{k-1}$, i.e.,

$$
C_{a_{1}}:=\sum_{\substack{a_{2}, a_{3}, \ldots, a_{k}=1 \\ a_{i} \neq a_{j}}}^{n} \mathrm{~s}_{a_{1} a_{2} \cdots a_{k}}=0 \quad \forall a_{1}
$$

Consider the linear combination $C_{2}+C_{3}+\ldots+C_{n}$ and collect terms into two groups. The first contains all terms that involve the label 1 and the second is the rest. This gives

$$
C_{2}+C_{3}+\ldots+C_{n}=\left(\sum_{a_{2}, a_{3}, \ldots, a_{k}=1 ; a_{i} \neq a_{j}}^{n} \mathrm{~s}_{1 a_{2} \ldots a_{k}}\right)+n\left(\sum_{1<a_{1}<a_{2}<\cdots<a_{k} \leq n} \mathrm{~s}_{a_{1} a_{2} \ldots a_{n}}\right) .
$$

The first group of terms on the r.h.s. is nothing but $C_{1}$ while the second is (3.6). Since all $C_{i}=0$ this concludes the argument that (3.3) with the identification (3.4) defines a valid potential function $\mathcal{S}_{n-k}$.

Before closing this section note that this duality already shows that the standard scattering equations which are defined on the space of $n$ point in $\mathbb{C P}^{1}$ are also equations for $n$ points on $\mathbb{C P}^{n-3}$. This is yet another indication of the importance of filling in the gap for other projective spaces in between. 


\section{Tropical Grassmannians and higher- $k$ Feynman diagrams}

In this section we use a surprising connection between tropical Grassmannians, the space of kinematic invariants, and the singularities that can arise in computing amplitudes using the scattering equations.

The connection starts with the tropical $G(2, n)$ Grassmannian and the standard space of kinematic invariants, i.e., the $k=2$ case. In this section we follow the construction of Speyer and Sturmfels [23] and use their notation. In particular, they denote the tropical Grassmannian $G(k, n)$ as $\mathcal{G}_{k, n}^{\prime \prime \prime}$. The map connects the vertices of the tropical Grassmannians with all possible kinematics invariant that can be poles of a Feynman diagram in a $\phi^{3}$ theory. For example, $\mathcal{G}_{2,4}^{\prime \prime \prime}$ is given by three points. These correspond to $s, t, u$. For $\mathcal{G}_{2,5}^{\prime \prime \prime}$ one has 10 vertices. Each vertex is associated with a given $s_{i j}$. One more case is needed to reach some generality. Consider the case $\mathcal{G}_{2,6}^{\prime \prime \prime}$; its 25 vertices are in correspondence with $s_{i j}$ and $t_{i j k}=s_{i j}+s_{j k}+s_{i k}$. In general $\mathcal{G}_{2, n}^{\prime \prime \prime}$ is known to have $2^{n-1}-n-1$ vertices labeled by all ways of partitioning the set $[n]:=\{1,2, \ldots, n\}$ into two sets $A, B$ with $|A|>1$ and $|B|>1$.

The analogy goes even beyond the structure of vertices. The edges of $\mathcal{G}_{2, n}^{\prime \prime \prime}$ can be mapped to all possible pairs of consistent poles or factorization of an amplitude. In other words, there is an edge between the vertices $\{A, B\}$ and $\left\{A^{\prime}, B^{\prime}\right\}$ if either $A \subset A^{\prime}$, or $A \subset B^{\prime}$ or $B \subset A^{\prime}$ or $B \subset B^{\prime}$.

Likewise the correspondence continues all the way to the facets for $\mathcal{G}_{2, n}^{\prime \prime \prime}$. As it turns out, the tropical Grassmannian $\mathcal{G}_{2, n}^{\prime \prime \prime}$ has $(2 n-5)$ !! facets which is precisely the number of all possible Feynman diagrams in $\phi^{3}$ theory. ${ }^{1}$

Motivated by this and by the careful study of $\mathcal{G}_{3,6}^{\prime \prime \prime}$ done in [23] we propose to extend the analogy to $k=3$ kinematics, biadjoint scalar amplitudes, and the corresponding generalization of Feynman diagrams.

Let us review the $\mathcal{G}_{3,6}^{\prime \prime \prime}$ results of [23] to exhibit the surprising connection with the objects found up to now and then use it to give a prescription for the computation of the most complicated of the biadjoint scalar amplitudes, i.e., $m_{6}^{(3)}(\mathbb{I} \mid \mathbb{I})$.

The main result of [23] is that $\mathcal{G}_{3,6}^{\prime \prime \prime}$ consists of 65 vertices, 550 edges, 1395 triangles and 1035 tetrahedra. In order to explain the structure of the vertices one introduces a basis of 20 unit vectors for $\left.\mathbb{R}^{(} \begin{array}{l}6 \\ 3\end{array}\right)$ labeled by three distinct and unordered indices $e_{i j k}$ that take values in $\{1,2, \ldots, 6\}$. The set of these 20 vectors is denoted as $E$. The space is then modded out by the image of the linear function $\phi: \mathbb{R}^{6} \rightarrow \mathbb{R}^{6}\left(\begin{array}{l}6 \\ 3\end{array}\right)$, given by

$$
\phi\left(w_{1}, \ldots, w_{6}\right)=\sum_{\{i, j, k\} \in E} e_{i j k}\left(w_{i}+w_{j}+w_{k}\right)=\sum_{i=1}^{6} w_{i} \sum_{\substack{j<k \\ j, k \neq i}} e_{i j k} .
$$

Setting the image of this function to zero accounts for imposing momentum conservation, thus we can identify the set $E$ with $\left\{s_{i j k}\right\}$ in this work. Moreover, for each 4 -subset of labels one defines [23]

$$
f_{i j k l}=e_{i j k}+e_{j k l}+e_{k l i}+e_{l i j} .
$$

\footnotetext{
${ }^{1}$ Connections between tropical geometry and scattering amplitudes in string theory were previously studied by Tourkine in [25].
} 
The set of such 15 vectors is denoted by $F$. Clearly, these correspond to $t_{i j k l}$. Even more surprising is the fact that Speyer and Sturmfels consider objects with six labels

$$
g_{i_{1} i_{2} i_{3} i_{4} i_{5} i_{6}}=f_{i_{1} i_{2} i_{3} i_{4}}+e_{i_{3} i_{4} i_{5}}+e_{i_{3} i_{4} i_{6}},
$$

which map to our $R$ and $\tilde{R}$. The set of these 30 vectors is $G$. The collection $E \cup F \cup G$ has 65 vectors and represent the vertices. From now on, we will not distinguish between the vertices and their corresponding kinematic invariants.

The next step is to define edges. There are six classes of edges whose definition can be read off from [23]. For instance, the first class is "EE" and is given by pairs of the type $\left\{\mathrm{s}_{a b c}, \mathrm{~s}_{a d e}\right\}$ or complementary labels as in $\left\{\mathrm{s}_{a b c}, \mathrm{~s}_{d e f}\right\}$. Inspection of the previous amplitudes reveals that only these combinations appear in $m_{6}^{(3)}(\alpha \mid \beta)$ ! This means we are interested in the set of facets as these will correspond to the new $k=3$ Feynman diagrams.

Tetrahedral facets are sets of four vertices $\left\{v_{1}, v_{2}, v_{3}, v_{4}\right\} \subset E$ with each pair $\left\{v_{i}, v_{j}\right\}$ being an edge. Speyer and Sturmfels find that the 990 tetrahedra split into six classes and 15 four-simplices. The six classes are given by all possible relabellings of the following representatives:

\begin{tabular}{|c|c|}
\hline EEEE & $\left\{\mathrm{s}_{123}, \mathrm{~s}_{345}, \mathrm{~s}_{561}, \mathrm{~s}_{246}\right\}$ \\
\hline EEFF1 & $\left\{\mathrm{s}_{123}, \mathrm{~s}_{456}, \mathrm{t}_{1234}, \mathrm{t}_{3456}\right\}$ \\
\hline EEFF2 & $\left\{\mathrm{s}_{123}, \mathrm{~s}_{345}, \mathrm{t}_{3456}, \mathrm{t}_{1236}\right\}$ \\
\hline EFFG & $\left\{\mathrm{s}_{345}, \mathrm{t}_{1256}, \mathrm{t}_{3456}, R_{34,12,56}\right\}$ \\
\hline EEEG & $\left\{\mathrm{s}_{123}, \mathrm{~s}_{561}, \mathrm{~s}_{345}, R_{23,45,61}\right\}$ \\
\hline EEFG & $\left\{\mathrm{s}_{123}, \mathrm{t}_{3456}, \mathrm{~s}_{345}, R_{34,12,56}\right\}$ \\
\hline
\end{tabular}

The remaining 15 four-simplices are permutations of labels of the facet

$$
\left\{\mathrm{t}_{1234}, \mathrm{t}_{3456}, \mathrm{t}_{5612}, R_{12,34,56}, R_{12,56,34}\right\} \text {. }
$$

This is nothing but the $\Theta$ diagram defined in (2.30). As explained by the authors, tetrahedra contained in these do not correspond to facets. These tetrahedra precisely correspond to the individual terms after splitting $\Theta$ using (2.29). This is the reason why they always appear combined in our previous examples.

Now we are ready to give a prescription for a combinatorial computation of $m_{6}^{(3)}(\alpha \mid \beta)$. We start by listing all possible vertices of $\mathcal{G}_{3,6}^{\prime \prime \prime}$ consistent with the planar ordering. This gives the list

$$
L(\mathbb{I})=\left\{R=R_{12,34,56}, R_{23,45,61}, \tilde{R}=R_{34,12,56}, R_{45,23,61}, \mathrm{~s}_{123}, \ldots, \mathrm{s}_{612}, \mathrm{t}_{1234}, \ldots, \mathrm{t}_{6123}\right\},
$$

of sixteen elements (by ... we denote cyclic shifts). Among the $\left(\begin{array}{c}12 \\ 4\end{array}\right)$ four-element sets of $L(\mathbb{I})$, we simply pick the ones that correspond to facets of $\mathcal{G}_{3,6}^{\prime \prime \prime}$ ! There are 46 such matches, each of these corresponds to a Feynman diagram of our amplitude. Finally, we append to the list the contribution from the $\Theta$ diagram and its cyclic shift $\bar{\Theta}$. This gives a list of 48 elements we denote by $J(\mathbb{I})$. The sum of all the elements of $J(\mathbb{I})$ corresponds to the 
$m_{6}^{(3)}(\mathbb{I} \mid \mathbb{I})$ amplitude, i.e.,

$$
m_{6}^{(3)}(\mathbb{I} \mid \mathbb{I})=\sum_{\Upsilon \in J(\mathbb{I})} \Upsilon
$$

Most of the terms were shown in section 2.2. However, there are new objects: the class EEEG gives contributions of the type $\frac{1}{\operatorname{sss} R}$ which only appear in the $m_{6}^{(3)}(\mathbb{I} \mid \mathbb{I})$ amplitude. Very nicely, as in the $k=2$ case, we can compute $m_{6}^{(3)}(\alpha \mid \beta)$ simply by considering the common Feynman diagrams of such orderings. In other words, denoting by $J(\alpha)$ and $J(\beta)$ the corresponding relabellings of $J(\mathbb{I})$, we have

$$
m_{6}^{(3)}(\alpha \mid \beta)=\sum_{\Upsilon \in J(\alpha) \cap J(\beta)} \Upsilon
$$

up to an overall sign. Finally, let us note that not all facets appear in the computation of $m_{6}^{(3)}(\mathbb{I} \mid \mathbb{I})$. This indicates that more general integrands are needed. For example, one can check that the EEEE facet $\left\{\mathbf{s}_{123}, \mathbf{s}_{345}, \mathrm{~s}_{561}, \mathrm{~s}_{246}\right\}$ never corresponds to a planar ordering. In fact, it is obtained by integrating

$$
\int \frac{d \mu_{3,6}}{(|123||345||561||246|)^{2}|234||456||612||153|}=\frac{1}{\mathrm{~s}_{123} \mathrm{~s}_{345} \mathrm{~s}_{561} \mathrm{~s}_{246}} .
$$

We leave the complete study of these correspondences to future work. However, before ending this section it is worth mentioning some of the results known in the mathematical literature which can help in the exploration of $k=3$ Feynman diagrams and $k=3$ amplitudes. In 2008, Herrmann et al. [26] revisited the tropical Grassmannian $G(3,6)$ and carefully studied the tropical Grassmannian $G(3,7)$. In their work they computed all rays that define the corresponding spaces and the combinations that make up the facets. Recall that rays (or vertices when considering the intersection with a unit sphere) are in bijection with the kinematic invariants that make "propagators" while the facets are proposed to correspond to the new $k=3$ Feynman diagrams. All the data collected in [26] is posted on the webpage: https://www.uni-math.gwdg.de/jensen/Research/G3_7/grassmann3_7.html.

Let us explain in more detail how to translate the tropical Grassmannian $G(3,7)$ data on the webpage to physics. The first important object to consider is the table of rays labeled $R$-vector. This is a list of 721 Plücker vectors. Each entry gives the coefficients of a vector in $\mathbb{R}^{35}$ in the basis $e_{i j k}$ ordered lexicographically. For example, the first entry is

$$
(0,0,0,0,0,0,0,0,0,0,0,0,0,0,0,0,0,0,0,0,0,0,0,0,0,0,0,0,0,0,0,0,0,0,1)
$$

and corresponds to the ray generated by $e_{567}$. The translation into physics is simply given again by identifying $e_{i j k}$ with $\mathrm{s}_{i j k}$.

As a second example take the element labelled 70 (or 71 in the list)

$$
(0,0,0,0,0,0,0,0,0,0,0,0,0,0,1,0,0,0,0,0,0,0,0,0,1,0,0,0,0,0,1,0,0,1,1)
$$

which corresponds to $e_{167}+e_{267}+e_{367}+e_{467}+e_{567}$ and translates to $\mathrm{s}_{167}+\mathrm{s}_{267}+\mathrm{s}_{367}+\mathbf{s}_{467}+$ $\mathrm{s}_{567}$. Using momentum conservation this also equals the more familiar invariant $t_{12345}$. 
The table of 721 rays is separated into six classes. We have translated all classes of rays into physical language and found that

\begin{tabular}{|c|c|}
\hline$[0,34]$ & $\mathrm{s}_{123}$ \\
\hline$[35,69]$ & $\mathrm{t}_{1234}$ \\
\hline$[70,90]$ & $\mathrm{t}_{12345}$ \\
\hline$[91,300]$ & $\mathrm{t}_{12345}+\mathrm{s}_{456}+\mathrm{s}_{457}$ \\
\hline$[301,615]$ & $\mathrm{t}_{1234}+\mathrm{t}_{1256}+\mathrm{s}_{127}$ \\
\hline$[616,720]$ & $\mathrm{t}_{12345}+t_{34567}+\mathrm{t}_{56712}$ \\
\hline
\end{tabular}

where the first column gives the labels of the first and last elements in the class while the second column is one of the representatives from which the others are obtained by permutations.

Finally, we can discuss the facets which are presented in the first table of the webpage and which can be read from the column labeled "Rays". As expected for $k=3$ and $n=7$ each Feynman diagram must consist of six propagators. Here each element in the list contains six rays. Consider for example the first row. The facet is given by rays $(0,7,14,18,25,27)$. From our table above it is easy to see that this facet only contains propagators of the form $s_{i j k}$ and therefore it is in the EEEEEE class in the notation used in this section. More explicitly, this facet then maps to the $k=3$ Feynman diagram

$$
(0,7,14,18,25,27) \rightarrow \frac{1}{\mathrm{~s}_{567} \mathrm{~s}_{347} \mathrm{~s}_{246} \mathrm{~s}_{235} \mathrm{~s}_{145} \mathrm{~s}_{136}} .
$$

\section{Matrix kinematics and MHV sectors}

The generalized kinematic invariants $\mathbf{s}_{a b c}$ have been treated as abstract objects so far. In this section we explore a generalization of the notion of momentum vectors that gives rise to a special class of kinematic invariants $\mathbf{s}_{a b c}$ analogous to four-dimensional kinematics when $k=2$. Moreover, on the support of these kinematics one can identify at least four analytic solutions of the scattering equations for all multiplicity, which are analogous to the four-dimensional MHV and $\overline{\mathrm{MHV}}$ solutions [27-29].

Let us start discussing the case of generic $k$ and then specialize to $k=3$. To each particle we associate two $k$-dimensional complex vectors $\lambda_{\alpha}^{(a)}$ and $\tilde{\lambda}_{\dot{\alpha}}^{(a)}$ which generalize the well-known spinor-helicity variables. Here the indices $\alpha, \dot{\alpha} \in\{1,2, \ldots, k\}$ transform under two copies of $\operatorname{SL}(k, \mathbb{C})$. Using these two vectors one can construct a $k \times k$ matrix $\mathbb{K}_{\alpha \dot{\alpha}}^{(a)}=\lambda_{\alpha}^{(a)} \tilde{\lambda}_{\dot{\alpha}}^{(a)}$. The redundancy $\operatorname{GL}(1) \subset \operatorname{SL}(k, \mathbb{C}) \times \operatorname{SL}(k, \mathbb{C})$ in this definition is the direct analog of the little group action, thus we can think of each $\lambda_{\alpha}^{(a)}$ and $\tilde{\lambda}_{\dot{\alpha}}^{(a)}$ as living in a projective space $\mathbb{C P}^{k-1}$.

The matrix $\mathbb{K}_{\alpha \dot{\alpha}}^{(a)}$ has rank one and therefore its determinant vanishes. Moreover, any linear combination of fewer than $k$ such matrices has rank smaller than $k$ and therefore vanishing determinant. This motivates the following definition

$$
\mathrm{s}_{a_{1} a_{2} \cdots a_{k}}:=\operatorname{det}\left(\mathbb{K}^{\left(a_{1}\right)}+\mathbb{K}^{\left(a_{2}\right)}+\cdots+\mathbb{K}^{\left(a_{k}\right)}\right) .
$$


With this definition it is clear that $\mathrm{s}_{a_{1} a_{2} \cdots a_{k}}$ is completely symmetric in its indices. Moreover, if any label repeats the invariant vanishes, i.e., $\mathrm{s}_{a_{1} a_{2} \cdots a_{k-2} b b}=0$. Furthermore, for $B=\left\{b_{1}, \ldots, b_{j}\right\}$ with $j>k$, the Cauchy-Binet theorem states that the determinant decomposes as ${ }^{2}$

$$
\begin{aligned}
\mathrm{t}_{B}:=\operatorname{det}\left(\mathbb{K}^{\left(b_{1}\right)}+\mathbb{K}^{\left(b_{2}\right)}+\cdots+\mathbb{K}^{\left(b_{j}\right)}\right) & =\sum_{\left\{a_{1}, \ldots, a_{k}\right\} \subset B} \operatorname{det}\left(\mathbb{K}^{\left(a_{1}\right)}+\mathbb{K}^{\left(a_{2}\right)}+\cdots+\mathbb{K}^{\left(a_{k}\right)}\right) \\
& =\sum_{\left\{a_{1}, \ldots, a_{k}\right\} \subset B} \mathrm{~s}_{a_{1} \cdots a_{k}} .
\end{aligned}
$$

Hence for $k=3$ the object $\mathrm{t}_{a b c d}$ can be identified as the l.h.s. . The $k=2$ analogy can actually be taken further as one can write

$$
\mathrm{s}_{a_{1} a_{2} \cdots a_{k}}=\left\langle a_{1} \ldots a_{k}\right\rangle\left[a_{1} \ldots a_{k}\right],
$$

where $\left\langle a_{1} \ldots a_{k}\right\rangle$ is the determinant of a $k \times k$ matrix of elements $\Lambda_{\alpha}^{i}=\lambda_{\alpha}^{\left(a_{i}\right)}$, and analogously $\left[a_{1} \ldots a_{k}\right]$ is the determinant of the matrix of elements $\tilde{\Lambda}_{\dot{\alpha}}^{i}=\tilde{\lambda}_{\dot{\alpha}}^{\left(a_{i}\right)}$. This can be seen by writing the argument of (5.1) as $\Lambda \tilde{\Lambda}^{T}$. Formula (5.3) also makes explicit the fact that each $\mathrm{s}_{a_{1} \cdots a_{k}}$ is linear in the $\mathbb{K}^{(a)}$ 's.

So far the invariants built from (5.1) satisfy properties analogous to the $k=2$ case. Let us then impose the generalized momentum conservation constraints (1.6). First note that adding all the $n$ conditions gives

$$
0=\sum_{a_{1}<\cdots<a_{k}} \mathrm{~s}_{a_{1} \cdots a_{k}}=\operatorname{det}\left(\mathbb{K}^{(1)}+\cdots+\mathbb{K}^{(n)}\right)
$$

by (5.2). Denoting the sum of all momenta by $Q=\sum_{i=1}^{n} \mathbb{K}^{(i)}$, this condition is the fact that $Q$ is of rank at most $k-1$, or, that $Q$ is the sum of at most $k-1$ rank-1 matrices:

$$
Q=\mathbb{Q}^{(1)}+\ldots+\mathbb{Q}^{(k-1)}, \quad \mathbb{Q}_{\alpha \dot{\alpha}}^{(i)}=q_{\alpha}^{(i)} \tilde{q}_{\dot{\alpha}}^{(i)} .
$$

We can write this as $\sum_{a=1}^{n} \lambda^{(a)} \tilde{\lambda}^{(a)}=-\sum_{i=1}^{k-1} q^{(i)} \tilde{q}^{(i)}$ and insert it into the conditions (1.6) to obtain

$$
\begin{aligned}
0 & =\sum_{a_{2}<\cdots<a_{k}}\left\langle a_{1} \cdots a_{k}\right\rangle\left[a_{1} \cdots a_{k}\right] \\
& =\left\langle a_{1} q^{(1)} \cdots q^{(k-1)}\right\rangle\left[a_{1} q^{(1)} \cdots q^{(k-1)}\right], \quad \forall a_{1} .
\end{aligned}
$$

To solve these conditions one can assume without loss of generality that

$$
0=\left\langle b q^{(1)} \cdots q^{(k-1)}\right\rangle, \quad b=1, \ldots,\left\lceil\frac{n}{2}\right\rceil,
$$

i.e., we have $\left\lceil\frac{n}{2}\right\rceil$ vectors living in a $k-1$ plane, where $\left\lceil\frac{n}{2}\right\rceil$ is the ceiling function. But in the cases we are studying ( $n \geq 3$ for $k=2$ and $n \geq 5$ for $k=3$ ) we have $\left\lceil\frac{n}{2}\right\rceil>k-1$. This

\footnotetext{
${ }^{2}$ A proof of this is obtained by regarding each $\mathbb{K}^{(a)}=\lambda^{(a)} \wedge \tilde{\lambda}^{(a)}$ as a two-form in $k+k$ dimensions. The determinant of any two-form $\omega$ is the coefficient of the top form $\omega^{\wedge k}:=\frac{1}{k !} \omega \wedge \ldots \wedge \omega$. Hence we can identify $s_{a_{1} \ldots a_{k}}$ with $\left(\sum_{i} \mathbb{K}^{\left(a_{i}\right)}\right)^{\wedge k}=\mathbb{K}^{\left(a_{1}\right)} \wedge \ldots \wedge \mathbb{K}^{\left(a_{k}\right)}$ and $t_{B}$ with $\left(\sum_{i} \mathbb{K}^{\left(b_{i}\right)}\right)^{\wedge k}$. The result (5.2) follows after expanding $t_{B}$. This also motivates the formula (5.3) and the subsequent manipulations.
} 
means that either the $\left\lceil\frac{n}{2}\right\rceil$ vectors $\lambda^{(b)}$ are degenerate, which would make the kinematic invariants $\mathrm{s}_{b_{1} \cdots b_{k}}$ vanish, or the $k-1$ vectors $q^{(i)}$ are degenerate, which would decrease the rank of $Q$ to $k-2$. As the first case corresponds to singular kinematics we focus on the second setup, which without loss of generality is obtained by writing (5.5) with

$$
\mathbb{Q}^{(k-1)}=0 .
$$

For $k=2$ this sets $Q=0$ and recovers the standard momentum conservation condition. Specializing to $k=3$, generalized momentum conservation now reads

$$
\mathbb{K}^{(1)}+\mathbb{K}^{(2)}+\cdots+\mathbb{K}^{(n)}=Q=q \tilde{q},
$$

where we call $q=q^{(1)}$ and $\tilde{q}=\tilde{q}^{(1)}$. At the level of the invariants we can use the familiar manipulations of the $k=2$ case, for instance, for $k=3, n=6$ we can write

$$
\mathrm{t}_{d e f g}=\mathrm{s}_{a b Q}=\sum_{c=1}^{6} \mathrm{~s}_{a b c}
$$

which is (2.38).

\subsection{Analytic solutions to the scattering equations}

In the $k=2$ case it is well known that solutions of the scattering equations split into $n-3$ sectors $[30,31]$. The $d$-th sector can be associated with two maps $\rho, \tilde{\rho}: \mathbb{C P}^{1} \rightarrow \mathbb{C P}^{1}$ of degrees $d$ and $n-2-d$ respectively. For $d=1$ and $d=n-3$ the maps are linear and the corresponding solutions are said to be in the MHV and $\overline{\mathrm{MHV}}$ sectors.

Here we provide evidence of the existence of such sector decomposition for higher $k$. We do this by constructing four analytic solutions which are present at any multiplicity for $k=3$. In particular this proves the existence of solutions to our scattering equations. The first two solutions lie in the direct analog of MHV and $\overline{\mathrm{MHV}}$ sectors and are easy to construct for any $k$. The other two are particular for $k=3$ as all the points in $\mathbb{C P}^{2}$ are found to lie on a conic. As they can also be identified as MHV-like solutions under a Veronese action, we will refer to them as $\mathrm{MHV}_{q}$ solutions. For $X(3,5)$ the $\mathrm{MHV}_{q}\left(\overline{\mathrm{MHV}_{q}}\right)$ sector coincides with the MHV $(\overline{\mathrm{MHV}})$ one. In particular this implies that there are only two solutions to the scattering equations at five points instead of four.

Let us first discuss the MHV solutions, the $\overline{\mathrm{MHV}}$ case being obtained by exchanging $\lambda_{\alpha} \leftrightarrow \tilde{\lambda}_{\dot{\alpha}}$. A degree-one map $\rho: \mathbb{C P}^{k-1} \rightarrow \mathbb{C P}^{k-1}$ can be written as $\rho(\sigma)=G \sigma$, where $G$ can be set to the identity by means of a $\operatorname{GL}(k, \mathbb{C})$ transformation in $X(k, n)$. Setting $k=3$, we adopt inhomogeneous coordinates by putting $\sigma=t(1, x, y)$. This means we can obtain our MHV solution simply as $\lambda^{(a)}=\sigma_{a}$, i.e.,

$$
x_{a}=\frac{\lambda_{2}^{(a)}}{\lambda_{1}^{(a)}}, \quad y_{a}=\frac{\lambda_{3}^{(a)}}{\lambda_{1}^{(a)}},
$$

where we have also solved the scale as $t_{a}=\lambda_{1}^{(a)}$. Inserting $\langle a b c\rangle=t_{a} t_{b} t_{c}(a b c)$ into the scattering equations gives

$$
\sum_{b, c} \frac{\langle a b c\rangle[a b c]}{(a b c)} x_{b c}=t_{a} \sum_{b, c} t_{b} t_{c} x_{b c}[a b c]=t_{a} \sum_{b, c}\langle r b c\rangle[a b c], \quad r=(0,0,1),
$$


and the analogous one for the $y$ coordinates. Momentum conservation (5.9) turns the last expression into $\langle r q q\rangle[a q q]=0$. This proves $(5.11)$ is a solution. Note finally that the scale $t_{a}$ drops in the cross-ratio combinations, which are also $\mathrm{GL}(k, \mathbb{C})$ invariant,

$$
\frac{\langle a b f\rangle\langle c d f\rangle}{\langle a d f\rangle\langle c b f\rangle}=\frac{(a b f)(c d f)}{(a d f)(c b f)} .
$$

This feature will have a nice analog for the $\mathrm{MHV}_{q}$ solutions, which we now introduce. For $k=3$ we define such solutions as the ones lying on a conic in $\mathbb{C P}^{2}$. As there is always a conic passing through five points, this explains why the $\mathrm{MHV}_{q}$ sector is contained in the MHV ones for $X(3,5)$. For $n>5$ one needs to set additional conditions so that the $n$ points lie on the conic defined by any five of them. Such conditions, i.e., the conic equations, can be stated as

$$
\frac{(a b f)(c d f)}{(a d f)(c b f)}=\frac{(a b g)(c d g)}{(a d g)(c b g)}, \quad f, g \neq a, b, c, d
$$

(this is an equation for $\sigma_{g}$ if $\left\{\sigma_{a}, \sigma_{b}, \sigma_{c}, \sigma_{d}, \sigma_{f}\right\}$ are considered fixed). It can be shown that these conditions are enough to arrange, by means of a $\mathrm{GL}(k, \mathbb{C}) \times\left(\mathbb{C}^{*}\right)^{n}$ transformation, any element of $X(3, n)$ into the Veronese form [32]. Such form is defined as $y_{a}=x_{a}^{2}$ in inhomogeneous coordinates. Under this parametrization $(a b c)=x_{a b} x_{b c} x_{c a}$ and the scattering equations become

$$
0=\sum_{b, c \neq a} \frac{\langle a b c\rangle[a b c]}{x_{a b} x_{a c}}, \quad 0=\sum_{b \neq a} \frac{\langle a b q\rangle[a b q]}{x_{a b}} \quad \forall a .
$$

As the objects $\langle a b q\rangle$ and $[a b q]$ can be identified with the standard $k=2$ brackets $\langle a b\rangle$ and $[a b]$ (for instance by choosing a frame where $q=(0,0,1)$ ), we recognize in the second set of conditions the standard scattering equations over $\mathbb{C P}^{1}$. As before, we know it admits two MHV-type solutions, which can be stated in a covariant form as

$$
x_{a}=\left\{\begin{array}{lll}
\frac{\langle a X q\rangle}{\langle a Y q\rangle} & \text { for } & \mathrm{MHV}_{q}, \\
\frac{[a X q]}{[a Y q]} & \text { for } & \overline{M H V}_{q},
\end{array}\right.
$$

where $X, Y$ are two reference vectors parametrizing the $\mathrm{SL}(2, \mathbb{C})$ redundancy of (5.15). As it turns out only these two solutions of the scattering equations are also solutions to the first set of conditions in (5.15). To see that such condition holds, let us take the $\mathrm{MHV}_{q}$ solution and use the Schouten identity to write $x_{a b}=\frac{\langle a b q\rangle\langle X Y q\rangle}{\langle a Y q\rangle\langle b Y q\rangle}$ so that

$$
\begin{aligned}
\sum_{b, c \neq a} \frac{\langle a b c\rangle[a b c]}{x_{a b} x_{a c}} & =\frac{\langle a Y q\rangle^{2}}{\langle X Y q\rangle^{2}} \sum_{b, c \neq a} \frac{\langle b Y q\rangle\langle c Y q\rangle}{\langle a b q\rangle\langle a c q\rangle}\langle a b c\rangle[a b c] \\
& =\frac{\langle a Y q\rangle}{\langle X Y q\rangle^{2}} \sum_{b, c \neq a} \frac{\langle b Y q\rangle\langle c Y q\rangle}{\langle a b q\rangle\langle a c q\rangle}(\langle a Y c\rangle\langle a b q\rangle-\langle a Y b\rangle\langle a c q\rangle)[a b c] \\
& =2 \frac{\langle a Y q\rangle}{\langle X Y q\rangle^{2}} \sum_{b, c \neq a} \frac{\langle b Y q\rangle\langle c Y q\rangle}{\langle a c q\rangle}\langle a Y c\rangle[a b c]=0,
\end{aligned}
$$


where the sum over $b$ again vanishes due to momentum conservation. Thus we have found two new solutions which lie on a conic given by (5.14). In fact, the cross-ratio in (5.13) now becomes $\frac{x_{a b} x_{c d}}{x_{a d} x_{c b}}$ so that we can write

$$
\frac{\langle a b q\rangle\langle c d q\rangle}{\langle a d q\rangle\langle c b q\rangle}=\frac{(a b f)(c d f)}{(a d f)(c b f)} \quad \forall f,
$$

which is a $\mathrm{GL}(k, \mathbb{C})$-invariant statement.

\section{$6 \quad$ Positive kinematics}

Motivated by the work of Kalousios [33], Zhang and two of the authors found a subregion of the $n(n-3) / 2$-dimensional space of kinematic invariants $s_{a b}$ where all $(n-3)$ ! solutions to the standard scattering equations, i.e., $k=2$, are real [24]. Moreover, the equations had the interpretation of the equilibrium points of a potential describing interacting particles on the interval $[0,1]$. This is easy to see by singling out three particles, $A, B, C$ and using $\operatorname{SL}(2, \mathbb{C})$ to set $x_{A}=0, x_{B}=1$ and $x_{C}=\infty$. The potential function is then ${ }^{3}$

$$
\operatorname{Re}\left(\mathcal{S}_{2}\right)=\sum_{a=1}^{n-3}\left(s_{A a} \log \left|x_{a}\right|+s_{B a} \log \left|1-x_{a}\right|\right)+\sum_{1 \leq a<b \leq n-3} s_{a b} \log \left|x_{a}-x_{b}\right| .
$$

Letting all $s_{A a}, s_{B a}, s_{a b}$ be positive gives rise to a system of $n-3$ particles on an interval, where all particles repel each other and are also repelled from the boundaries of the interval at $x=0$ and $x=1$. This region is called $\mathcal{K}_{n}^{+}$. Note that the choice $s_{A a}, s_{B a}, s_{a b}>0$ is possible because they form a basis of the $n(n-3) / 2$-dimensional kinematic space. ${ }^{4}$

In this section we generalize the notion of positive kinematics to $k=3$ and discuss some of the new features that appear. In the same way as for $k=2$, we find for $n<7$ that all solutions are real and give rise to a very elegant and pictorial derivation of the number of solutions.

We start by selecting four particles $A, B, C, D$ to be fixed by the action of $\mathrm{SL}(3, \mathbb{C})$. This time two particles, say $C, D$, can be sent to infinity by setting their homogeneous coordinates to $(0,1,0)$ and $(0,0,1)$ respectively. The other two are chosen to be, in inhomogeneous coordinates, at the origin and at $(1,1)$ on the plane $(x, y) \in \mathbb{R}^{2}$, i.e., $A$ has homogeneous coordinates $(1,0,0)$ while $B$ has $(1,1,1)$.

Clearly the $n=4$ case is trivial as all four particles are gauge fixed. Since interactions in the potential function are controlled by the determinants $|a b c|$ a given particle is not directly sensitive to the location of any other particle but only to the lines defined by any pair of particles. In order to find the analog of the positive region in this case let us again consider the potential function

$$
\operatorname{Re}\left(\mathcal{S}_{3}\right)=\sum_{\substack{a=1 \\ I<J \in\{A, B, C, D\}}}^{n-4} s_{I J a} \log |I J a|+\sum_{\substack{1 \leq a<b \leq n-4 \\ I \in\{A, B, C, D\}}} s_{I a b} \log |I a b|+\sum_{1 \leq a<b<c \leq n-4} s_{a b c} \log |a b c| .
$$

\footnotetext{
${ }^{3}$ Notice that taking the real value $\operatorname{Re}\left(\mathcal{S}_{k}\right)$ instead of $\mathcal{S}_{k}$ does not affect the positions of the critical points if all $s_{a_{1} a_{2} \cdots a_{k}}$ are real.

${ }^{4}$ For a mathematical perspective, see, e.g., [34].
} 

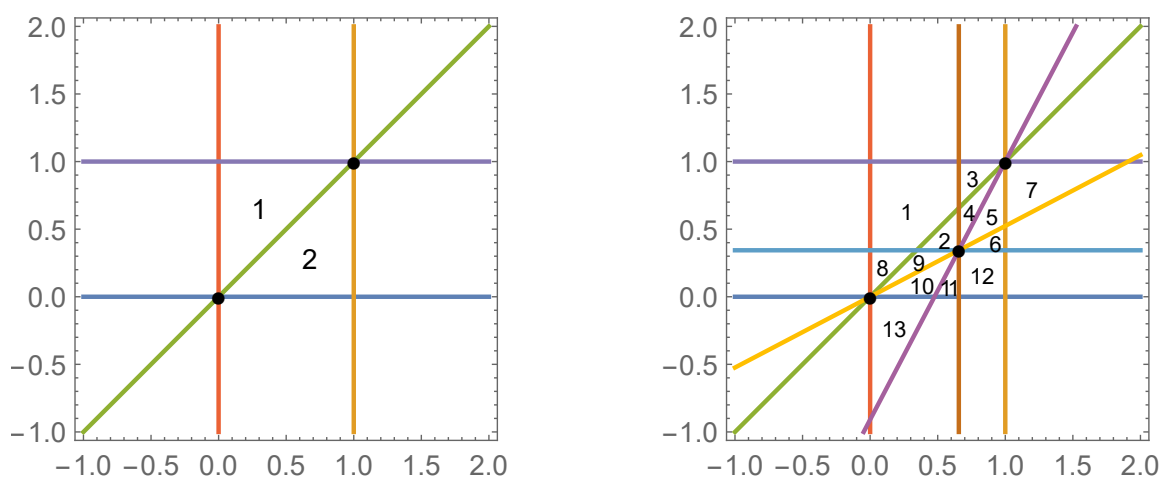

Figure 1. Left: five lines seen by the free particles when $n=5$. Points $A$ and $B$ are shown as black dots while $C$ and $D$ are at infinity. Out of all 12 chambers, only two are bounded and are labeled. Right: once the point 1 is fixed to a location in the bounded chamber 2, it generates four new lines which then increase the total number of chambers to 31. Only 13 chambers are bounded and are explicitly labeled.

Our first approach to the $k=3$ positive region $\mathcal{K}_{3, n}^{+}$is then to ask all invariants that explicitly appear in (6.2) to be positive. This is possible because once again they form a basis for the space of $k=3$ kinematic invariants. More explicitly, the only constrains on kinematic invariants are determined by the $n$ conditions which generalize $k=2$ momentum conservation. These are linear equations for the $n$ variables $s_{A B C}, s_{B C D}, s_{A C D}, s_{A B D}$ and $s_{C D a}$ for $1 \leq a \leq n-4$.

Now we are ready to study the dynamics generated by the potential $\mathcal{S}_{3}$. Consider $n=5$. Since only one point is free to move we use $x, y$ for its coordinates. Here the potential is

$$
\operatorname{Re}\left(\mathcal{S}_{3}\right)=s_{A B 1} \log |x-y|+s_{A C 1} \log |y|+s_{A D 1} \log |x|+s_{B C 1} \log |1-y|+s_{B D 1} \log |1-x| .
$$

Since all coefficients are positive it is possible to understand the dynamics as that of a particle in $\mathbb{R}^{2}$ which is repelled from five lines. The lines are, in the order in which they appear in the potential, $x=y, y=0, x=0, y=1$, and $x=1$. This is shown in figure 1 (left). Since critical points of the potential correspond to equilibrium points, it is clear that they can only lie in the bounded chambers of the space, i.e., those with finite area. Note that the five lines divide the plane into 12 chambers. Ten of them are unbounded and two are bounded. Therefore there are only two possible places for particle 1 to be located, and since we know from section 2.2 that there are exactly two solutions to the scattering equations, they have to lie in these two chambers.

Much more interesting is the $n=6$ case. Here we use the soft limit approach in order to more clearly understand the dynamics. We assume that $\left|s_{i j 2}\right| \ll\left|s_{k l m}\right|$. This means that one can effectively solve the scattering equations in steps. First we find solutions for particle 1. As before, we find two of them in the two bounded chambers. Once a solution is picked, we study the dynamics of the second particle, assumed to be soft. Let us choose the lower chamber (chamber 2 in figure 1). Particle 2 now interacts with the same five lines given above and also with four new lines. The new lines all pass through the point 1 . One 

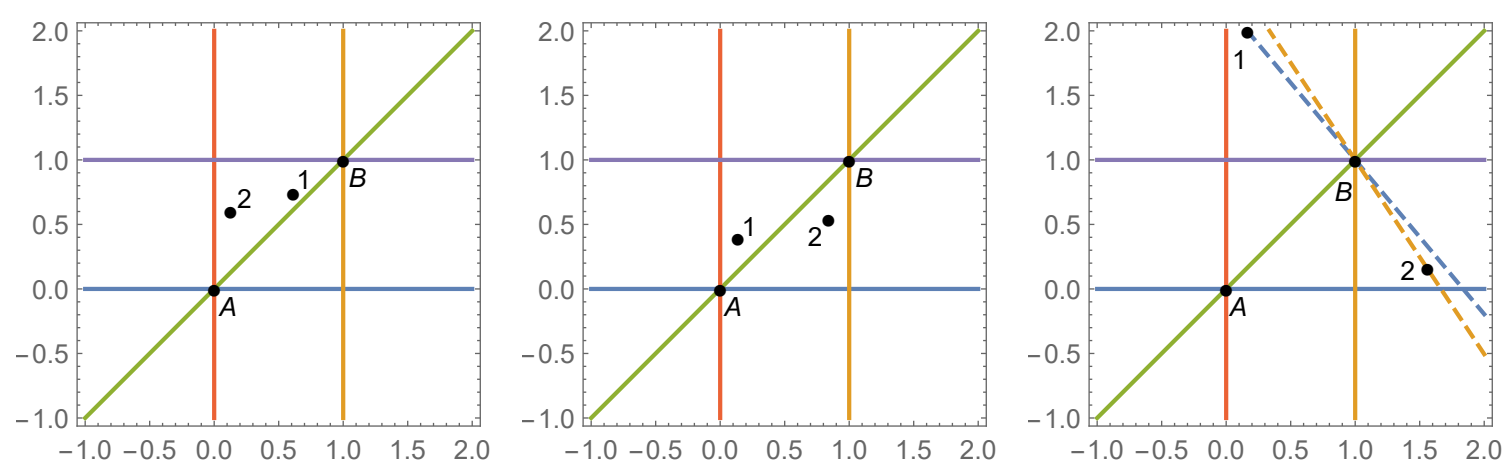

Figure 2. Three families of configurations of solutions on $\mathbb{R P}^{2}$ for generic positive kinematics. Left: solutions in the same chamber. Center: solutions in distinct chambers. Right: solutions outside of the square $[0,1]^{2}$.

is parallel to the $x$ axis, another to the $y$ axis, while the remaining two pass one through $A$ at $(0,0)$ and the other through $B$ at $(1,1)$. These lines are depicted in figure 1 (right).

Clearly, particle 2 can only find equilibrium points on bounded chambers. It is easy to count 31 chambers in total with 13 bounded and 18 unbounded. On the figure we have explicitly labeled all 13 bounded chambers. This can be repeated again for the second equilibrium point of particle 1 thus obtaining another set of 13 critical points, summing to 26. This saturates the total number of solutions (see appendix A) and hence the above argument describes all of them.

These pictures also reveal that our first attempt at defining a positive region cannot be a completely connected one. The reason is that according to our definition, one can smoothly change the kinematics invariants from the soft region $\left|s_{i j 2}\right| \ll\left|s_{k l m}\right|$ to a new one where the roles of 1 and 2 are reversed, i.e., $\left|s_{i j 1}\right| \ll\left|s_{k l m}\right|$. This leads to a problem as the solutions where particle 2 is placed on region 7 or region 13 in the figure disappear as soon as particle 1 becomes soft and 2 hard.

This means that there must be new singularities that separate the two soft regions. These are not of the form $s_{i j k}=0$ or $t_{i j k l}=0$ since they all have definite sign in the positive region. This puzzle is resolved by the novel $k=3$ singularities found in the biadjoint amplitudes in section 2.2 and denoted by $R$ and $\tilde{R}$.

It is possible to see that some of the $R$ 's and $\tilde{R}$ 's do not have definite sign and hence can become zero as we move from one soft region to the next.

In fact, on kinematics that is positive but generic, i.e., not near any soft region, we find that the 26 solutions split in classes shown in figure 2 . In 16 of them points 1 and 2 are located on the same $n=4$ bounded chamber while 6 have 1 and 2 on two distinct bounded chambers. The remaining four have both 1 and 2 in unbounded chambers. This is possible because one particle generates a new bounded chamber for the other! In the examples given in figure 2 (right), point 2 generates a bounded chamber for 1 with the line $2-B$, while particle 1 generates a bounded chamber for 2 with the line $1-B$, both indicated by dashed lines.

Before ending this section let us mention that one can be tempted to continue the construction of chambers for more particles and count the number of solutions to the $k=3$ 
scattering equations in this way. More explicitly, placing particle 2 in any of the 13 bounded chambers generated by particle 1 gives rise to a diagram with 42 bounded chambers. This implies that there must be at least $2 \times 13 \times 42$ solutions for $n=7$. In appendix B we carefully study soft limits directly from the scattering equations and find a closed formula for the bounded regions and also explain why for $n>6$ and $k=3$ these numbers are strict lower bounds.

\section{Discussion}

In this work we have initiated the study of a natural generalization of scattering equations to moduli spaces of points on $\mathbb{C P}^{k-1}$. We have only scratched the surface of what seems to be a vast subject. The duality explained in section 3 between the space of $n$ points on $\mathbb{C P}^{k-1}$ and that of $n$ points on $\mathbb{C P}^{n-k-1}$ shows that the standard $k=2$ scattering equations, which are at the heart of the CHY formalism, already imply the existence of the $k=n-2$ scattering equations. Moreover, the standard Mandelstam invariants $s_{a b}$ give rise to $k=n-2$ kinematic invariants. In this work we have filled the gap between these two end points by studying intermediate values of $k$.

We found that the all the elements needed to define generalized CHY amplitudes are also present for any $k$ and gave the explicit formulation of "biadjoint amplitudes". The first non-trivial case is six points on $\mathbb{C P}^{2}$, i.e., the scattering equations relating the space of kinematic invariants $\mathrm{s}_{a b c}$ to $X(3,6)$. Explicit computations of biajoint amplitudes led to the discovery of new kinematic poles $R$ and $\tilde{R}$ in addition to the expected s and t poles. A very pressing question is the explicit computation for $n>6$ and $k=3$. A direct computation for seven particles seems technically very challenging as the number of solutions jumps from 26 for $n=6$ to more than a thousand for $n=7$. However, it is well known that in the $k=2$ case, a variety of techniques have been developed that allow the evaluation of CHY formulae without actually solving the scattering equations, see, e.g., [35-37].

One of the early successes of the CHY formalism was the direct connection between biadjoint scalar amplitudes and the Kawai-Lewellen-Tye construction and the simple derivation of the Bern-Carrasco-Johansson basis of color ordered amplitudes. These developments rely on the fact that the basis of Parke-Taylor functions on $X(2, n)$ has size $(n-3)$ ! when evaluated on the solutions of the scattering equations which happens to be the same as the number of solutions. It is clear that a generalization of these construction to higher $k$ is very desirable. At this point there is evidence that Parke-Taylor functions might not provide the most general basis of functions needed for $2<k<n-2$.

Another natural question is the generalization of the notion of Feynman diagrams for $k>2$. When $n=6$ and $k=3$ we have made a proposal based on the computations of section 2 and on the surprising connection to tropical Grassmannians explored and used in section 4. Tropical Grassmannians are polyhedral complexes with what seems to be a very direct connection to the space of kinematic invariants. The vertices of the tropical Grassmannian are in bijection with all possible poles in a $\phi^{3}$ theory. Moreover, the facets are in bijection with all possible individual Feynman diagrams of the theory. We have found that the same is true for $k=3$ and $n=6$ for the vertices and kinematic poles. 
Using the natural assumptions that facets must also correspond to Feynman diagrams we developed a combinatorial computation of all biadjoint amplitudes by computing the most fundamental one, $m_{6}^{(3)}(\mathbb{I} \mid \mathbb{I})$. We noted that one class, the EEEE class, of $k=3$ Feynman diagrams did not contribute to $m_{6}^{(3)}(\mathbb{I} \mid \mathbb{I})$ and hence to any of the biadjoint amplitudes. This is strong evidence that for $k=3$ one has to go beyond Parke-Taylor factors as building blocks for the integrand.

Another piece of evidence for the need of more general integrands comes from the $k=3$ Schouten identity. In $k=2$ the Schouten identity

$$
(13)(24)=(12)(34)+(14)(23)
$$

gives rise what is known as the U(1)-decoupling identity among Parke-Taylor functions by dividing (7.1) by all the factors

$$
\frac{1}{(12)(23)(34)(41)}+\frac{1}{(14)(42)(23)(31)}+\frac{1}{(12)(24)(43)(31)}=0 .
$$

The $k=3$ Schouten identity reads

$$
(123)(456)+(341)(256)=(234)(156)+(412)(356) .
$$

In the same way as before this leads to the following 4-term identity

$$
\begin{aligned}
& \frac{1}{(341)(256)(234)(156)(412)(356)}+\frac{1}{(123)(456)(234)(156)(412)(356)}= \\
& \quad=\frac{1}{(412)(356)(123)(456)(341)(256)}+\frac{1}{(234)(156)(123)(456)(341)(256)} .
\end{aligned}
$$

None of these four terms are Parke-Taylor functions. However, it is possible to multiply (7.4) by a product of six factors, e.g., $(236)^{2}(145)^{2}(612)(345)$, such that each of the four terms are turned into the product of two Parke-Taylor functions but we take this still as an indication that more general functions are needed.

We also found an explicit realization of the $k$ space of kinematic invariants in terms of a generalization of the spinor-helicity formalism. Rank one $2 \times 2$ matrices are replaced by rank one $k \times k$ matrices and invariants are computed as determinants of sums of such matrices. Since the factorization, $s_{a b}=\langle a b\rangle[a b]$, that led to miraculous simplifications for $k=2$ also happens for any $k>2$, it is tempting to suggest that there is a generalization of constructions such as BCFW recursion, superamplitudes, etc. One immediate challenge is the definition of the notion of helicity and polarization vectors. We leave these fascinating questions for future research.

Another interesting connection made for $k=2$ is that between the CHY formalism, string theory [6, 38-42], Z-theory [43-45], and ambitwistor strings [46, 47]. It is clear that a generalization of $Z$-theory integrals is possible for $k>2$ and natural to expect that on a subregion of the positive kinematics defined in section 6 , where appropriate $R$ 's are positive, such integrals should exist.

Finally, there are two more very useful constructions known for $k=2$ which can greatly impact the ability of solving $k>2$ scattering equations. The first is obtaining what 
is known as the polynomial form of the scattering equations first constructed by Dolan and Goddard in [9]. These polynomial form allows a faster and more stable numerical search as well as a direct way of counting the number of solutions using Bezout's theorem since the equations increase their degree in steps of one. The second development is the identification of an integrable system with the scattering equations for a particular set of kinematics invariant. This was done by Kalousios and led to a connection to Jacobi polynomials [33]. We started the exploration of such kind of kinematics in section 6 but leave the search for an integrable system for future work. Either one of these construction would help in completing the counting of solutions started in section 6 and in appendix B where an attempt to follow the soft limit approach led only to lower bounds.

\section{Acknowledgments}

We would like to thank F. Borges, D. Garcia, S. He, J. Rojas, S. Yusim, and Y. Zhang for useful discussions. N.E. would like to thank Perimeter Institute for their support and hospitality while this work was initiated. A.G. thanks CONICYT for financial support via the project 21151647. This research was supported in part by Perimeter Institute for Theoretical Physics. Research at Perimeter Institute is supported by the Government of Canada through the Department of Innovation, Science and Economic Development Canada and by the Province of Ontario through the Ministry of Research, Innovation and Science.

\section{A Euler characteristic of $X(3,6)$}

In this appendix we give a derivation of the fact that scattering equations on $X(3,6)$ have 26 solutions. As explained in [6], CHY formulae can be understood as intersection numbers of twisted differential forms, which in our case belong to the cohomology group

$$
H^{4}\left(X(3,6), d \mathcal{S}_{3} \wedge\right):=\frac{\text { holomorphic 4-forms on } X(3,6)}{d \mathcal{S}_{3} \wedge(\text { holomorphic } 3 \text {-forms on } X(3,6))},
$$

where $\mathcal{S}_{3}$ is the Morse function (1.5) for $n=6$. In other words, it is the space of 4 -forms modulo anything proportional to the scattering equations. This realization allows us compute the dimension of the space (A.1), and hence also the number of solutions $\mathcal{N}_{6}^{(3)}$ of the scattering equations (2.2), purely topologically as the Euler characteristic of $X(3,6)$. More precisely, under the assumption that the kinematics is generic (so that all other twisted cohomology groups vanish), one can show that

$$
\mathcal{N}_{6}^{(3)}=(-1)^{4} \chi(X(3,6))
$$

using Morse theory, see, e.g., [6,34]. We can use a chart in which punctures $\{1,2,3,4\}$ are held fixed, such that $X(3,6)$ can be written as the complement of the projective variety

$$
V:=\bigcup_{\substack{1 \leq i<j<k \leq 6 \\ k \geq 5}}\{|i j k|=0\}
$$


in $\left(\mathbb{C P}^{2}\right)^{2}$. Using the inclusion-exclusion principle we can write the Euler characteristic as

$$
\begin{aligned}
\chi(X(3,6)) & =\chi\left(\left(\mathbb{C P}^{2}\right)^{2}\right)-\chi(V) \\
& =3 \times 3-\chi(V),
\end{aligned}
$$

where we used $\chi\left(\mathbb{C P}^{2}\right)=3$. The remaining contribution $\chi(V)$ can be evaluated using the algebraic geometry system Macaulay2 [48] with the package CharacteristicClasses.m2 [49, $50]$ as follows. Let $\mathrm{R}$ be the coordinate ring of $\left(\mathbb{C P}^{2}\right)^{2}$ (say over $\mathbb{Z} / p \mathbb{Z}$ for $p=32749$ ) with coordinates $r_{-} i$ for $i=0,1, \ldots, 5$, and $I$ be the ideal generated by vanishing of all the relevant maximal minors of the matrix

$$
\left(\begin{array}{llllll}
1 & 0 & 0 & 1 & r \_0 & r_{-} 3 \\
0 & 1 & 0 & 1 & r \_1 & r_{-} 4 \\
0 & 0 & 1 & 1 & r \_2 & r_{-5}
\end{array}\right) .
$$

Then the Euler characteristic in (A.4) can be computed using the following script:

load "CharacteristicClasses.m2";

$\mathrm{R}=$ MultiProjCoordRing(ZZ/32749, symbol $r,\{2,2\})$;

$I=i d e a l\left(r_{-} 0 * r_{-} 1 * r_{-} 2 * r_{-} 3 * r_{-} 4 * r_{-} 5 *\left(r_{-} 0-r_{-} 1\right) *\left(r_{-} 0-r_{-} 2\right) *\left(r_{-} 1-r_{-} 2\right) *\left(r_{-} 3-r_{-} 4\right)\right.$

$*\left(r_{-} 3-r_{-} 5\right) *\left(r_{-} 4-r_{-} 5\right) *\left(r_{-} 0 * r_{-} 4-r_{-} 1 * r_{-} 3\right) *\left(r_{-} 0 * r_{-} 5-r_{-} 2 * r_{-} 3\right)$

$\left.*\left(r_{-} 1 * r_{-} 5-r_{-} 2 * r_{-} 4\right) *\left(r_{-} 0 *\left(r_{-} 4-r_{-} 5\right)+r_{-} 1 *\left(r_{-} 5-r_{-} 3\right)+r_{-} 2 *\left(r_{-} 3-r_{-} 4\right)\right)\right)$;

$3 * 3-E u l e r(I)$

The output of this computation is 26 , which by (A.2) gives the number of solutions of scattering equations, $\mathcal{N}_{6}^{(3)}=26$. Similar computation for $X(3,5)$ yields $\mathcal{N}_{5}^{(3)}=2$.

\section{B Soft limits and numbers of solutions}

One of the most basic questions about the scattering equations is the number of solutions. It is well-known that the $k=2$ scattering equations for $n$ particles have $(n-3)$ ! solutions [1]. Let us review one technique for proving it and then try and apply it for $k=3$. The idea is to approach what is known as the soft-limit region for the $n^{\text {th }}$ particle. This is done by writing all invariants of the form $\mathrm{s}_{a n}$ as $\tau \hat{\mathrm{s}}_{a n}$ in the limit when $\tau \rightarrow 0$. In the limit the $n^{\text {th }}$ particle drops from the first $n-1$ scattering equations which become those for a system of $n-1$ particles. The $n^{\text {th }}$ equation is proportional to $\tau$ even for finite $\tau$ and therefore it fixes the location of $x_{n}$. Assuming that the system for $n-1$ has been solved and $\mathcal{N}_{n-1}^{(2)}$ solutions have been found, each such solution $x_{a,(I)}$ give rise to an equation for $x_{n}$

$$
\sum_{a=1}^{n-1} \frac{\hat{\mathbf{s}}_{a n}}{x_{a,(I)}-x_{n}}=0 .
$$


It is easy to show that this leads to $n-3$ solutions for $x_{n}$. Therefore $\mathcal{N}_{n}^{(2)}=(n-3) \mathcal{N}_{n-1}^{(2)}$. For $n=4$ (B.1) is all there is after fixing three points and therefore $\mathcal{N}_{4}^{(2)}=1$. This leads to the expected result $\mathcal{N}_{n}^{(2)}=(n-3)$ !.

Strictly speaking, the soft argument only leads to a lower bound on the number of solutions as one has to prove that when taking $\tau=0$ in the first $n-1$ equations one is allowed to drop terms that depend on particle $n$. For instance, it could happen that there are solutions where $x_{n}-x_{a} \sim \mathcal{O}(\tau)$ and hence the term $s_{a n} /\left(x_{n}-x_{a}\right)$ cannot be dropped. Indeed this happens when collinear limits, $s_{a n} \rightarrow 0$, are taken and it is well-known that solutions split into two classes: singular and regular. Regular solutions are those for which the term $s_{a n} /\left(x_{n}-x_{a}\right)$ can be dropped. This means that the soft limit argument is only guarantee to count the regular solutions. It turns out that when $k=2$ there are no singular solutions in the soft limit. Unfortunately, this is not the case when $k>3$.

Here we repeat the same argument for $k=3$, one takes the "soft" limit $\mathbf{s}_{a b n}=\tau \hat{\mathbf{s}}_{a b n}$ with $\tau \rightarrow 0$. We will remove all dependence on particle $n$ in the equations that defined the $(n-1)$ system, i.e., we will only compute the number of regular solutions.

Assuming that the system for $(n-1)$ particles has been solved, one finds for each solution $x_{a,(I)}, y_{a,(I)}$ two equations

$$
\sum_{1 \leq b<c \leq n-1} \frac{\mathrm{s}_{n b c}\left(x_{b,(I)}-x_{c,(I)}\right)}{\langle n b c\rangle_{(I)}}=0, \quad \sum_{1 \leq b<c \leq n-1} \frac{\mathrm{s}_{n b c}\left(y_{b,(I)}-y_{c,(I)}\right)}{\langle n b c\rangle_{(I)}}=0 .
$$

We are only interested in counting the number of solutions to these "soft" equations. In order to analyze these equations one has to rewrite them as the ratio of two polynomials

$$
\frac{P_{A}\left(x_{n}, y_{n}\right)}{\prod_{1 \leq b<c \leq n-1}\langle n b c\rangle_{(I)}}=0, \quad \frac{P_{B}\left(x_{n}, y_{n}\right)}{\prod_{1 \leq b<c \leq n-1}\langle n b c\rangle_{(I)}}=0 .
$$

Counting the number of solutions to the system $P_{A}\left(x_{n}, y_{n}\right)=P_{B}\left(x_{n}, y_{n}\right)=0$ is harder than in the $k=2$ case for two reasons.

In order to understand the first, recall that when dealing with a single polynomial in one variable, as in $k=2$, the degree of the polynomial equation coming from the numerator of (B.1) directly gives the number of solutions. The first complication for $k=3$ arises from the fact that for two polynomials in two variables, $\left(x_{n}, y_{n}\right)$, Bezout's theorem only gives an upper bound for the number of solutions at finite locations. The bound is the product of the degrees of the two polynomials.

The second difficulty comes from the fact that the system $P_{A}\left(x_{n}, y_{n}\right)=P_{B}\left(x_{n}, y_{n}\right)=0$ has solutions which are not solutions to the original equations (B.2). Such solutions come from where two factors in the denominator of (B.3) vanish. To see this note that if the poles are approached as $\epsilon \rightarrow 0$, then the original equations (B.2) diverge as $1 / \epsilon$ while the denominators in (B.3) diverge as $1 / \epsilon^{2}$. This shows that both $P_{A}$ and $P_{B}$ must vanish as $\epsilon \rightarrow 0$. This means that we have to remove these spurious solutions from Bezout's bound.

Very nicely, the final formula turns out to be simple

$$
\operatorname{Soft}_{n}^{(3)}=\frac{1}{8}(n-4)\left(n^{3}-6 n^{2}+11 n-14\right) .
$$


Now it is tempting to construct the number of solutions as the product of all soft factors up to the number of particles of interest, i.e.

$$
\mathcal{N}_{n}^{(3) \text { :naive }}=\prod_{m=5}^{n} \operatorname{Soft}_{m}^{(3)} .
$$

However, as discussed above, the soft limit computation only captures regular solutions. We will now show why when $k=3$ there must also be singular solutions as well and leave their enumeration for future work.

Note that $\mathcal{N}_{n}^{(3) \text { :naive }}$ seems to contain a factor of $(n-4)$ !. However, since the polynomial

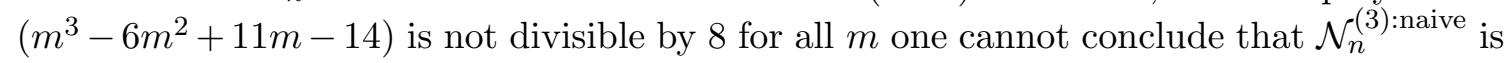
divisible by $(n-4)$ !. In fact already for $n=8$, this naive number of solutions is not divisible by $(n-4)$ ! which is a contradiction with the $(3, n) \Leftrightarrow(n-3, n)$ duality discussed in section 3 .

To see this more explicitly, note that when $n=7$ one can repeat the same soft argument for $k=4$. Starting from $n=6, k=2$ which has six solutions one finds that so does $n=6$, $k=4$ by the duality in section 3 . An explicit computation reveals that the soft equations for the seventh particle have 192 solutions. This means that the number of solutions must be at least $6 \times 192=1152$. This is 60 more than $\mathcal{N}_{7}^{(3) \text { :naive }}=2 \times 13 \times 42=1092$.

\section{Numerical solution to the $X(3,6)$ scattering equations}

The $k=3, n=6$ scattering equations have 26 solutions. For general kinematic invariants the 26 solutions cannot be found analytically. In this appendix we give and explicit procedure for finding all 26 solutions to arbitrarily-high precision for a kinematic point in the positive region. The procedure involves finding approximate solutions with low precision, which we call seeds, using a numerical search and then each seed is used as an input in the Mathematica's FindRoot function set up to high precision.

One the kinematic points we used is given by the following values of the independent invariants

$$
\begin{aligned}
& \mathrm{s}_{123}=139, \quad \mathrm{~s}_{124}=179, \quad \mathrm{~s}_{125}=223, \quad \mathrm{~s}_{126}=257, \quad \mathrm{~s}_{134}=199, \\
& \mathrm{~s}_{135}=241, \quad \mathrm{~s}_{136}=281, \quad \mathrm{~s}_{145}=271, \quad \mathrm{~s}_{146}=313, \quad \mathrm{~s}_{156}=-2103, \\
& \mathrm{~s}_{234}=227, \quad \mathrm{~s}_{235}=263, \quad \mathrm{~s}_{236}=307, \quad \mathrm{~s}_{245}=283, \quad \mathrm{~s}_{246}=337, \\
& \mathrm{~s}_{256}=-2215, \quad \mathrm{~s}_{345}=-2025, \quad \mathrm{~s}_{346}=-2239, \quad \mathrm{~s}_{356}=2607, \quad \mathrm{~s}_{456}=2455 .
\end{aligned}
$$

It is easy to verify that these invariants satisfy momentum conservation and that none of the possible poles of $k=3$ Feynman diagrams vanish.

We use the same gauge fixing as in section 2 :

$$
\left(\begin{array}{llllll}
1 & 1 & 1 & 1 & 1 & 1 \\
0 & 1 & 0 & 1 & x_{5} & x_{6} \\
0 & 0 & 1 & 1 & y_{5} & y_{6}
\end{array}\right)
$$


The seeds for the 26 solutions are

$$
\begin{array}{llll}
x_{5} \rightarrow-1.81336 & x_{6} \rightarrow 2.63087 & y_{5} \rightarrow 2.72136 & y_{6} \rightarrow-1.06766 \\
x_{5} \rightarrow 2.44712 & x_{6} \rightarrow-1.79167 & y_{5} \rightarrow-0.903713 & y_{6} \rightarrow 2.69014 \\
x_{5} \rightarrow 0.534746 & x_{6} \rightarrow 0.537102 & y_{5} \rightarrow-2.70181 & y_{6} \rightarrow-0.201572 \\
x_{5} \rightarrow 0.534421 & x_{6} \rightarrow 0.528164 & y_{5} \rightarrow-0.175607 & y_{6} \rightarrow-2.36545 \\
x_{5} \rightarrow 1.8782 & x_{6} \rightarrow-3.70997 & y_{5} \rightarrow 1.82661 & y_{6} \rightarrow-2.60442 \\
x_{5} \rightarrow 0.50317 & x_{6} \rightarrow 0.470842 & y_{5} \rightarrow 0.374444 & y_{6} \rightarrow-1.38027 \\
x_{5} \rightarrow 1.04874 & x_{6} \rightarrow 1.55304 & y_{5} \rightarrow 0.24654 & y_{6} \rightarrow-0.45775 \\
x_{5} \rightarrow 0.475295 & x_{6} \rightarrow 0.503754 & y_{5} \rightarrow-1.54168 & y_{6} \rightarrow 0.365724 \\
x_{5} \rightarrow 0.237534 & x_{6} \rightarrow 0.836314 & y_{5} \rightarrow 1.70422 & y_{6} \rightarrow 1.57173 \\
x_{5} \rightarrow-2.92233 & x_{6} \rightarrow 1.88376 & y_{5} \rightarrow-1.97545 & y_{6} \rightarrow 1.82657 \\
x_{5} \rightarrow 1.01926 & x_{6} \rightarrow 1.16625 & y_{5} \rightarrow 0.661554 & y_{6} \rightarrow 0.389534 \\
x_{5} \rightarrow 1.00838 & x_{6} \rightarrow 0.917236 & y_{5} \rightarrow 1.08258 & y_{6} \rightarrow 0.909572 \\
x_{5} \rightarrow 1.51702 & x_{6} \rightarrow 1.05038 & y_{5} \rightarrow-0.425664 & y_{6} \rightarrow 0.257 \\
x_{5} \rightarrow 0.386006 & x_{6} \rightarrow 0.653472 & y_{5} \rightarrow 0.712486 & y_{6} \rightarrow 0.731654 \\
x_{5} \rightarrow 0.500766 & x_{6} \rightarrow 0.49462 & y_{5} \rightarrow 0.420314 & y_{6} \rightarrow 0.142516 \\
x_{5} \rightarrow-0.00949757 & x_{6} \rightarrow 0.094872 & y_{5} \rightarrow 1.11668 & y_{6} \rightarrow 0.896041 \\
x_{5} \rightarrow 0.9293 & x_{6} \rightarrow 1.00989 & y_{5} \rightarrow 0.92216 & y_{6} \rightarrow 1.10112 \\
x_{5} \rightarrow-0.697705 & x_{6} \rightarrow-0.0509408 & y_{5} \rightarrow-0.583952 & y_{6} \rightarrow 0.266517 \\
x_{5} \rightarrow 0.841712 & x_{6} \rightarrow 0.242035 & y_{5} \rightarrow 1.58999 & y_{6} \rightarrow 1.68593 \\
x_{5} \rightarrow 0.0782592 & x_{6} \rightarrow-0.0105854 & y_{5} \rightarrow 0.913617 & y_{6} \rightarrow 1.14256 \\
x_{5} \rightarrow-0.0198028 & x_{6} \rightarrow-0.188344 & y_{5} \rightarrow 0.63874 & y_{6} \rightarrow 0.367487 \\
x_{5} \rightarrow 1.17891 & x_{6} \rightarrow 1.02194 & y_{5} \rightarrow 0.372119 & y_{6} \rightarrow 0.662613 \\
x_{5} \rightarrow-0.203209 & x_{6} \rightarrow-0.0221714 & y_{5} \rightarrow 0.351966 & y_{6} \rightarrow 0.642902 \\
x_{5} \rightarrow-0.0501271 & x_{6} \rightarrow-0.755866 & y_{5} \rightarrow 0.254807 & y_{6} \rightarrow-0.636579 \\
x_{5} \rightarrow 0.650306 & x_{6} \rightarrow 0.383491 & y_{5} \rightarrow 0.727378 & y_{6} \rightarrow 0.716881 \\
x_{5} \rightarrow 0.495514 & x_{6} \rightarrow 0.500968 & y_{5} \rightarrow 0.128367 & y_{6} \rightarrow 0.41208 \\
& & &
\end{array}
$$

Using the function FindRoot with four scattering equations as arguments and the seeds as initial conditions one can easily generate all 26 solutions to 500 digits of precision.

Using the high-precision solutions the $k=3$ biadjoint formulas can be evaluated. The numerical result, which is guaranteed to be a rational number since the coefficients of all scattering equations are rational, can be easily turn into a rational number using the Mathematica function Rationalize.

Open Access. This article is distributed under the terms of the Creative Commons Attribution License (CC-BY 4.0), which permits any use, distribution and reproduction in any medium, provided the original author(s) and source are credited. 


\section{References}

[1] F. Cachazo, S. He and E.Y. Yuan, Scattering equations and Kawai-Lewellen-Tye orthogonality, Phys. Rev. D 90 (2014) 065001 [arXiv:1306.6575] [INSPIRE].

[2] F. Cachazo, S. He and E.Y. Yuan, Scattering of massless particles in arbitrary dimensions, Phys. Rev. Lett. 113 (2014) 171601 [arXiv:1307.2199] [inSPIRE].

[3] F. Cachazo, S. He and E.Y. Yuan, Scattering of massless particles: scalars, gluons and gravitons, JHEP 07 (2014) 033 [arXiv:1309.0885] [INSPIRE].

[4] H. Kawai, D.C. Lewellen and S.-H. Henry Tye, A relation between tree amplitudes of closed and open strings, Nucl. Phys. B 269 (1986) 1 [INSPIRE].

[5] Z. Bern, J.J.M. Carrasco and H. Johansson, New relations for gauge-theory amplitudes, Phys. Rev. D 78 (2008) 085011 [arXiv: 0805.3993] [INSPIRE].

[6] S. Mizera, Scattering amplitudes from intersection theory, Phys. Rev. Lett. 120 (2018) 141602 [arXiv: 1711.00469] [INSPIRE].

[7] P. Mastrolia and S. Mizera, Feynman integrals and intersection theory, JHEP 02 (2019) 139 [arXiv: 1810.03818] [INSPIRE].

[8] H. Frellesvig et al., Decomposition of Feynman integrals on the maximal cut by intersection numbers, JHEP 05 (2019) 153 [arXiv: 1901.11510] [INSPIRE].

[9] L. Dolan and P. Goddard, The polynomial form of the scattering equations, JHEP 07 (2014) 029 [arXiv: 1402.7374] [INSPIRE].

[10] S.J. Parke and T.R. Taylor, An amplitude for $n$ gluon scattering, Phys. Rev. Lett. 56 (1986) 2459 [INSPIRE].

[11] N. Arkani-Hamed, J.L. Bourjaily, F. Cachazo, A.B. Goncharov, A. Postnikov and J. Trnka, Grassmannian geometry of scattering amplitudes, Cambridge University Press, Cambridge, U.K. (2016) [arXiv:1212.5605] [inSPIRE].

[12] F. Cachazo, N. Early, A. Guevara and S. Mizera, $\Delta$-algebra and scattering amplitudes, JHEP 02 (2019) 005 [arXiv: 1812.01168] [INSPIRE].

[13] S. Franco, D. Galloni, B. Penante and C. Wen, Non-planar on-shell diagrams, JHEP 06 (2015) 199 [arXiv: 1502.02034] [InSPIRE].

[14] J.L. Bourjaily, S. Franco, D. Galloni and C. Wen, Stratifying on-shell cluster varieties: the geometry of non-planar on-shell diagrams, JHEP 10 (2016) 003 [arXiv:1607.01781] [INSPIRE].

[15] M.M. Kapranov, Chow quotients of Grassmannians I, Adv. Soviet Math. 16 (1993) 29 [alg-geom/9210002].

[16] J. Sekiguchi, The versal deformation of the $E_{6}$-singularity and a family of cubic surfaces, $J$. Math. Soc. Jpn. 46 (1994) 355.

[17] J. Sekiguchi, Cross ratio varieties for root systems, Kyushu J. Math. 48 (1994) 123.

[18] J. Sekiguchi and M. Yoshida, $W\left(E_{6}\right)$-action on the configuration space of six lines on the real projective plane, Kyushu J. Math. 51 (1997) 297.

[19] J. Sekiguchi, Configurations of seven lines on the real projective plane and the root system of type $E_{7}$, J. Math. Soc. Jpn. 51 (1999) 987. 
[20] J. Sekiguchi, Cross ratio varieties for root systems II: the case of the root system of type $E_{7}$, Kyushu J. Math. 54 (2000) 7.

[21] S. Keel and J. Tevelev, Chow quotients of Grassmannians II, math.AG/0401159.

[22] S. Keel and J. Tevelev, Geometry of Chow quotients of Grassmannians, Duke Math. J. 134 (2006) 259.

[23] D. Speyer and B. Sturmfels, The tropical Grassmannian, Adv. Geom. 4 (2004) 389 [math.AG/0304218].

[24] F. Cachazo, S. Mizera and G. Zhang, Scattering equations: real solutions and particles on a line, JHEP 03 (2017) 151 [arXiv:1609.00008] [INSPIRE].

[25] P. Tourkine, Tropical amplitudes, Annales Henri Poincaré 18 (2017) 2199 [arXiv: 1309.3551] [INSPIRE].

[26] S. Herrmann, A. Jensen, M. Joswig and B. Sturmfels, How to draw tropical planes, Electron. J. Combinat. 16 (2009) 6 [arXiv:0808.2383].

[27] D.E. Roberts, Mathematical structure of dual amplitudes, Ph.D. thesis, Durham University, Durham, U.K. (1972) [INSPIRE].

[28] D.B. Fairlie and D.E. Roberts, Dual models without tachyons - a new approach, (1972) [INSPIRE].

[29] D.B. Fairlie, A coding of real null four-momenta into world-sheet coordinates, Adv. Math. Phys. 2009 (2009) 284689 [arXiv: 0805.2263] [INSPIRE].

[30] M. Spradlin and A. Volovich, From twistor string theory to recursion relations, Phys. Rev. D 80 (2009) 085022 [arXiv:0909.0229] [INSPIRE].

[31] F. Cachazo, S. He and E.Y. Yuan, Scattering in three dimensions from rational maps, JHEP 10 (2013) 141 [arXiv: 1306.2962] [INSPIRE].

[32] N. Arkani-Hamed, J. Bourjaily, F. Cachazo and J. Trnka, Unification of residues and Grassmannian dualities, JHEP 01 (2011) 049 [arXiv:0912.4912] [INSPIRE].

[33] C. Kalousios, Massless scattering at special kinematics as Jacobi polynomials, J. Phys. A 47 (2014) 215402 [arXiv: 1312.7743] [INSPIRE].

[34] K. Aomoto, On vanishing of cohomology attached to certain many valued meromorphic functions, J. Math. Soc. Jpn. 27 (1975) 248.

[35] C. Baadsgaard, N.E.J. Bjerrum-Bohr, J.L. Bourjaily and P.H. Damgaard, Integration rules for scattering equations, JHEP 09 (2015) 129 [arXiv:1506.06137] [INSPIRE].

[36] H. Gomez, $\Lambda$ scattering equations, JHEP 06 (2016) 101 [arXiv: 1604.05373] [INSPIRE].

[37] X. Gao, S. He and Y. Zhang, Labelled tree graphs, Feynman diagrams and disk integrals, JHEP 11 (2017) 144 [arXiv:1708.08701] [INSPIRE].

[38] H. Gomez and E.Y. Yuan, $N$-point tree-level scattering amplitude in the new Berkovits' string, JHEP 04 (2014) 046 [arXiv: 1312.5485] [INSPIRE].

[39] W. Siegel, Amplitudes for left-handed strings, arXiv:1512.02569 [INSPIRE].

[40] E. Casali and P. Tourkine, On the null origin of the ambitwistor string, JHEP 11 (2016) 036 [arXiv: 1606.05636] [INSPIRE]. 
[41] S. Mizera and G. Zhang, A string deformation of the Parke-Taylor factor, Phys. Rev. D 96 (2017) 066016 [arXiv: 1705.10323] [INSPIRE].

[42] E. Casali and P. Tourkine, Windings of twisted strings, Phys. Rev. D 97 (2018) 061902 [arXiv: 1710.01241] [INSPIRE].

[43] J.J.M. Carrasco, C.R. Mafra and O. Schlotterer, Abelian Z-theory: NLSM amplitudes and $\alpha^{\prime}$-corrections from the open string, JHEP 06 (2017) 093 [arXiv: 1608.02569] [INSPIRE].

[44] C.R. Mafra and O. Schlotterer, Non-Abelian Z-theory: Berends-Giele recursion for the $\alpha^{\prime}$-expansion of disk integrals, JHEP 01 (2017) 031 [arXiv: 1609.07078] [INSPIRE].

[45] J.J.M. Carrasco, C.R. Mafra and O. Schlotterer, Semi-Abelian Z-theory: NLSM+ $\phi^{3}$ from the open string, JHEP 08 (2017) 135 [arXiv:1612.06446] [INSPIRE].

[46] L. Mason and D. Skinner, Ambitwistor strings and the scattering equations, JHEP 07 (2014) 048 [arXiv: 1311.2564] [INSPIRE].

[47] Y. Geyer, Ambitwistor strings: worldsheet approaches to perturbative quantum field theories, Ph.D. thesis, Oxford U., Inst. Math., Oxford, U.K. (2016) [arXiv: 1610.04525] [INSPIRE].

[48] D.R. Grayson and M.E. Stillman, Macaulay2, a software system for research in algebraic geometry, http://www.math.uiuc.edu/Macaulay2/.

[49] C. Jost, Computing characteristic classes and the topological Euler characteristic of complex projective schemes, J. Softw. Alg. Geom. 7 (2015) 31.

[50] M. Helmer, Computing characteristic classes of subschemes of smooth toric varieties, J. Alg. 476 (2017) 548. 